\title{
Structural and Functional Consequences of the Weak Binding of Chlorin e6 to Bovine Rhodopsin
}

\author{
James Mitchell ${ }^{1}$, Naveena Yanamala ${ }^{2}$, Yi Lei Tan ${ }^{3}$, Eric E. Gardner ${ }^{2}$, Kalyan C. Tirupula ${ }^{2}$, Fernanda \\ Balem $^{2}$, Mordechai Sheves ${ }^{4}$, Daniel Nietlispach ${ }^{3}$ and Judith Klein-Seetharaman ${ }^{1,2^{*}}$
}

${ }^{1}$ Biomedical Sciences Division, Warwick Medical School, University of Warwick, Coventry, CV4 7AL, UK

${ }^{2}$ Dept. of Structural Biology, School of Medicine, University of Pittsburgh, Pittsburgh, PA 15260, USA

${ }^{3}$ Department of Biochemistry, 80 Tennis Court Road, University of Cambridge, Cambridge, CB2 1GA, UK

${ }^{4}$ Organic Chemistry Department, Weizmann Institute of Science, 234 Herzl Street, Rehovot 7610001, Israel

\section{Keywords:}

Allosteric modulator, porphyrin, chlorophyll-derivative, photosensitization, night vision, light activation, bovine rhodopsin, $\mathrm{G}$ protein coupled receptor

"Corresponding author's email: j.klein-seetharaman@warwick.ac.uk (Judith Klein-Seetharaman)

This article has been accepted for publication and undergone full peer review but has not been through the copyediting, typesetting, pagination and proofreading process, which may lead to differences between this version and the Version of Record. Please cite this article as doi: 10.1111/php.13074

This article is protected by copyright. All rights reserved. 


\section{ABSTRACT}

The chlorophyll-derivative chlorin e6 (Ce6) identified in the retinas of deep-sea ocean fish is proposed to play a functional role in red bioluminescence detection. Fluorescence and ${ }^{1} \mathrm{H}$ NMR spectroscopy studies with the bovine dim-light photoreceptor, rhodopsin, indicate that Ce6 weakly binds to it with $\mu \mathrm{M}$ affinity. Absorbance spectra prove that red light sensitivity enhancement is not brought about by a shift in the absorbance maximum of rhodopsin. ${ }^{19} \mathrm{~F}$ NMR experiments with samples where ${ }^{19} \mathrm{~F}$ labels are either placed at the cytoplasmic binding site or incorporated as fluorinated retinal, indicate that the cytoplasmic domain is highly perturbed by binding, while little to no changes are detected near the retinal. Binding of Ce6 also inhibits $\mathrm{G}$ protein activation. Chemical shift changes in ${ }^{1} \mathrm{H},{ }^{15} \mathrm{~N}$ NMR spectroscopy of ${ }^{15} \mathrm{~N}$-Trp labeled bovine rhodopsin reveal that Ce6 binding perturbs the entire structure. These results provide experimental evidence that $\mathrm{Ce} 6$ is an allosteric modulator of rhodopsin.

\section{INTRODUCTION}

Rhodopsin, the dim-light mammalian photoreceptor in rods, is a prototypical member of the opsin family, which is a large sub-group within the G protein coupled receptor (GPCR) family. GPCRs adopt the overall organization of a seven transmembrane helical bundle. All opsins covalently bind retinal, a vitamin A derivative, at the interface between the transmembrane and extracellular domains. Retinal binds to Lys-296 in bovine rhodopsin (Figure 1). Visual signal transduction is initiated by photon-induced isomerization of 11-cis retinal to all-trans-retinal. This event is sensed by the transmembrane domain, which undergoes a conformational change that results in the activated state, Metarhodopsin II (Meta II), via another intermediate, Meta I. Meta II - unlike dark state rhodopsin and Meta I - binds and activates the $G$ protein, transducin $\left(G_{t}\right)$, ultimately leading to receptor hyperpolarization. Signal desensitization is initiated by phosphorylation of the C-terminus of rhodopsin by rhodopsin kinase, followed by binding of arrestin, preventing further binding of $G_{t}$ to rhodopsin. In vitro, Meta II decays to form opsin and free retinal. The half-life of Meta II decay depends on the lipid environment, and is approximately 10 minutes in detergent solutions (1). In addition, in parallel with Meta II, there is transient formation of a storage form of rhodopsin, Meta III, from the Meta I state. The Meta III state of rhodopsin - unlike Meta II - has a protonated retinal Schiff base and decays into opsin and free retinal on longer time scales (2-6).

This article is protected by copyright. All rights reserved. 
Chlorophyll derivatives were found in the retinas of the deep-sea ocean fish Malacosteus niger, a type of dragon fish, and have been proposed to act as a photosensitizer extending its vision to red light (7-9). Table 1 summarizes the in vivo deep-sea fish rhodopsin experiments that led to their discovery. Although the hypothesis that Malacosteus niger "sees" with chlorophyll (7) was never experimentally validated, a chlorophyll derivative, chlorin e6 (Ce6) has been administered either orally or intravenously in rats and rabbits in order to test for night vision enhancement (10). The chemical structure of $\mathrm{Ce} 6$ is shown in Figure 2a. Ce6 localizes to the retina and retinal pigment epithelium, and a two-fold increase in electroretinographic b-wave amplitudes as a response to red and blue light was found, consistent with a potential night vision benefit (10).

Molecular support for the red-light sensitization effects came from studies carried out with preparations of the retina that contain the highest density of rhodopsin, the rod outer segments (ROS). Ce6 and several other porphyrin compounds were found to increase the bleaching rates of the retinal chromophore in rhodopsin by red light (11). It was hypothesized but not experimentally validated that energy transfer from $\mathrm{Ce} 6$ to retinal in rhodopsin, resembling that of light harvesting in photosynthesis, may be the basis for the photosensitizing effect $(8,11)$. Finally, Ce6 was found to enhance the thermal stability of rhodopsin in vitro (12). A combination of computational docking and experimental terahertz spectroscopy confirmed that $\mathrm{Ce} 6$ binds in the cytoplasmic domain of rhodopsin and allosterically excites long-range correlated fluctuations that connect distant parts of rhodopsin. Such long-range correlated fluctuations are conserved across the entire class A GPCR family (13).

To understand the functional consequences of the allosteric activation of evolutionarily conserved signaling pathways in the rhodopsin structure, we studied the binding of $\mathrm{Ce} 6$ to purified bovine rhodopsin. M. niger rhodopsin is $68.6 \%$ identical, and $91.2 \%$ similar to bovine rhodopsin (as aligned by the Needleman-Wunsch algorithm, using the BLOSUM similarity matrix $(14,15))$. Bovine rhodopsin has been thoroughly characterized using biophysical and biochemical approaches, and is therefore a good model system for Ce6 experiments. We determined the binding affinity of Ce6 for bovine rhodopsin to be low, in the $\mu \mathrm{M}$ range. We find that affinity and structural details of the interaction differ for dark-state, Meta II and opsin conformations, with preferred binding occurring in Meta II. ${ }^{19}$ F NMR data experimentally supports a cytoplasmic location of Ce6 binding, further supported by the observation that binding inhibits $G$ protein activation. NMR evidence suggests that Ce6 binding perturbs the equilibrium between light-activated and opsin states and modulates the rhodopsin structural globally, supporting Ce6's classification as an allosteric modulator of rhodopsin activation.

This article is protected by copyright. All rights reserved. 


\section{MATERIALS AND METHODS}

Materia/s. Unlabeled and ${ }^{19} \mathrm{~F}$ labeled rhodopsin were prepared from bovine retinae (W.A. Lawson Co., Lincoln, NE) as described (16). Rhodopsin reconstituted with fluororetinal was prepared from cultured HEK293 cells stably transfected to express bovine rhodopsin. These cells were obtained from Philip Reeves (School of Biological Sciences, University of Essex). 11-cis-Retinal was a gift from Rosalie Crouch (University of South Carolina and the National Eye Institute of the National Institutes of Health U.S. Public Health Services). 9-cis-Retinal was obtained from Sigma-Aldrich, St. Louis, MO. 9-cis-14-fluororetinal was prepared as previously described (17). Bovine rhodopsin was affinitypurified using the rhodopsin C-terminal antibody 1D4 (18) (University of British Columbia) coupled to Sepharose-4B (Sigma) and eluted using the nonapeptide (Genscript Limited, Hong Kong) corresponding to the C-terminal sequence of rhodopsin, the antibody epitope. Ce6 was obtained from Frontier Scientific, Logan, UT. The sources of all other reagents have been described (16). Illumination of the samples in all other experiments was carried out with a Fiber-Lite DC 950 regulated illuminator by Dolan-Jenner industries, unless otherwise stated.

Reconstitution with 14-fluororetinal. Rhodopsin was illuminated with a Schott KI 1500 light source with an open aperture, set to $3400 \mathrm{~K}$ and a $495 \mathrm{~nm}$ filter for 60 seconds. 14-fluororetinal was added to a five times excess to rhodopsin. It was found that reducing the temperature of reconstitution from $20^{\circ} \mathrm{C}$ to $4^{\circ} \mathrm{C}$ improved reconstitution yield from $29 \%$ to $43 \%$. Reconstitution was also carried out in the presence of 2:1 Ce6:rhodopsin, and yield was unaffected.

Absorbance spectroscopy. Bovine rhodopsin in $2 \mathrm{mM}$ sodium phosphate $\mathrm{pH} 6,0.05 \% \mathrm{DM}$ was diluted to $400 \mu \mathrm{L}$ at $\sim 1 \mu \mathrm{M}$, and a rhodopsin spectrum recorded. Ce6 was added from $100 \mathrm{mM}$ stock, or a dilution thereof, in DMSO so the volume added was between 1 and $4 \mu \mathrm{L}$, to the desired concentration. An initial spectrum was taken as well as a spectrum after each 30 second illumination through a $630 \mathrm{~nm}$ long-pass filter (HV Skan, UK), using a Schott KL1500 light source. To confirm no bleaching was due to ambient light, the same procedure was followed, but covering the end of the fibre-optic, and no bleaching occurred. After five minutes of red illumination, the $630 \mathrm{~nm}$ filter was exchanged for a $495 \mathrm{~nm}$ filter and the light source was set to $3400 \mathrm{~K}$, and the sample illuminated for 60 seconds for full bleaching of rhodopsin.

This article is protected by copyright. All rights reserved. 
After decomposition (see below) the $A_{500}$ of the $\mathrm{Ce} 6$ subtracted spectra at each time point was used to calculate the concentration of rhodopsin. The $A_{500}$ of the final, $495 \mathrm{~nm}$ illuminated spectrum was first subtracted, then the fraction remaining was calculated. The time course was fitted to an exponential decay curve $\left([\right.$ Rhodopsin $\left.]=A e^{-k t}\right)$. The rate constant $\left(\mathrm{k}, \min ^{-1}\right)$ was recorded, and plotted against the ratio of Ce6:rhodopsin, calculated from the $A_{665}$ and the $A_{500}$ respectively.

To measure rhodopsin dark noise, the cuvettes were in a water jacketed cell holder with the water bath set to the desired temperature. Spectra were recorded every 20 minutes for 420 minutes.

To decompose spectra, the rhodopsin spectrum was corrected for dilution due to addition of $\mathrm{Ce} 6$ by multiplying by $400 /\left(400+V_{\mathrm{Ce}}\right)$, where the volume before adding Ce6 was $400 \mu \mathrm{L}$, and $\mathrm{V}_{\mathrm{Ce}}$ is the volume of $\mathrm{Ce} 6$ added (in $\mu \mathrm{L}$ ). This was then subtracted from the initial Ce6 spectrum to isolate the Ce6 contribution. For each illuminated spectrum, the Ce6 spectrum was multiplied by the ratio of $A_{664}$ for that spectrum to the example $\mathrm{Ce} 6$ spectrum to subtract the $\mathrm{Ce} 6$ contribution and account for its bleaching. By using the Ce6 contribution acquired in this way, any differences in the spectrum of $\mathrm{Ce} 6$ in this sample from a free $\mathrm{Ce} 6$ spectrum, as have been observed when $\mathrm{Ce} 6$ has electrostatic interactions with cosolutes $(19,20)$ are eliminated.

Fluorescence spectroscopy. Binding of $\mathrm{Ce} 6$ or as a control Chl-a was quantified by measuring the fluorescence of $4 \mu \mathrm{g}$ of rhodopsin in $500 \mu \mathrm{l}$ of $2 \mathrm{mM}$ sodium phosphate $\mathrm{pH} 6,0.05 \% \mathrm{DM}$ as described (1). Ce6 was added prior to illumination from a $100 \mathrm{mM}$ DMSO stock solution or its dilutions, at the final concentrations indicated. The fluorescence measured at each concentration was corrected for Ce6 absorption using the Parker method $(21,22)$. The function used for correcting the data is

$$
F_{(\text {real })}=\left(F_{(\text {measured })}{ }^{\star} 2.303^{\star} A\right) /\left(1-10^{-A}\right)
$$

where $A$ is the absorbance of the sample at $295 \mathrm{~nm}$ (excitation) or $330 \mathrm{~nm}$ (emission). $F_{\text {(real) }}$ and $F_{\text {(measured) }}$ are the corrected and observed fluorescence values at each concentration of $\mathrm{Ce} 6$ or $\mathrm{Chl}-\mathrm{a}$ measured (21). The same correction was applied to dark and light samples, as the absorbance spectra at the excitation and emission wavelengths used did not change. For light-activating rhodopsin, the samples were illuminated with a $150 \mathrm{~W}$ fiber optic light (Fiber Lite A-200; DolanJenner, Woburn, MA) equipped with a > $495 \mathrm{~nm}$ long-pass filter for 30 seconds.

This article is protected by copyright. All rights reserved. 
To maintain the fluorescence samples under anaerobic conditions, an oxygen trap was used. 5 $\mathrm{mM}$ glucose, $0.1 \mathrm{mg} / \mathrm{ml}$ of glucose oxidase and $0.1 \mathrm{mg} / \mathrm{ml}$ of catalase were added to $500 \mu$ l solutions containing $0.5 \mu \mathrm{M}$ of rhodopsin and $5 \mu \mathrm{M}$ of Ce6. The samples were incubated for $5 \mathrm{~min}$ to achieve equilibrium prior to recording fluorescence spectra.

Binding Affinity Estimations. The binding affinities for the interaction were calculated with the onesite saturation ligand binding function using Sigmaplot 10.0 scientific graphing software.

NMR spectroscopy. All operations were carried out in the dark, unless otherwise stated.

${ }^{19} \mathrm{~F}$ NMR spectroscopy of bovine rhodopsin was performed as described previously (16). Onedimensional ${ }^{19} \mathrm{~F}$ NMR spectra were acquired on a $600 \mathrm{MHz}\left({ }^{1} \mathrm{H}\right)$ Bruker instrument with a ${ }^{19} \mathrm{~F},{ }^{1} \mathrm{H},{ }^{15} \mathrm{~N}$ TXO $5 \mathrm{~mm}$ triple gradient probe at $20^{\circ} \mathrm{C}$. Data acquisition and analysis was carried out using TopSpin Version 2.0 Software. The sample was locked on deuterium. The relaxation delay was $0.5 \mathrm{sec}$. Line broadening was $20 \mathrm{~Hz}$. An internal standard, TFA, was used as control or reference in each case. Each spectrum was acquired within 2 minutes on a $600 \mathrm{MHz}$ Bruker instrument, at $20{ }^{\circ} \mathrm{C}$. Each spectrum is an average of 105 scans. A line broadening of $10 \mathrm{~Hz}$ was used.

The $1 \mathrm{D}{ }^{1} \mathrm{H}$ selective excitation NMR spectra were recorded at a spectrometer ${ }^{1} \mathrm{H}$ frequency of 800 $\mathrm{MHz}$ at the Structural Biology Department, University of Pittsburgh using a Bruker spectrometer. The details of the acquisition of 1D selective excitation ${ }^{1} \mathrm{H}$ NMR spectra are as follows. As the samples contained detergent micelles, a selective excitation scheme with sculpting, using a double pulse field gradient spin echo sequence (DPFGSE) as described previously $(23,24)$ was used. Each spectrum was obtained after applying two hyperbolic secant shaped pulses of length $2.8 \mathrm{~ms}$ and $1.9 \mathrm{~ms}$, following a $90^{\circ}$ hard pulse of $9.9 \mu$ s at $0.5 \mathrm{~dB}$ over a spectral width of $35 \mathrm{ppm}$. A delay of 0.5 seconds between each scan was used. A total of 2048 scans were averaged to obtain the final spectrum. The total acquisition time was $\sim 29 \mathrm{~min}, 7 \mathrm{~min}$ and $2.2 \mathrm{~min}$ to acquire 2048, 512 and 160 scans, respectively. A line broadening of $0.3 \mathrm{~Hz}$ or $1 \mathrm{~Hz}$ was used to process the final spectra.

${ }^{1} \mathrm{H}-{ }^{15} \mathrm{~N}$ HSQC NMR spectra of $\alpha-\varepsilon^{-}{ }^{15} \mathrm{~N}$-tryptophan were obtained at a spectrometer frequency of $\sim 900 \mathrm{MHz}$ Bruker spectrometer. Data acquisition and analysis was then carried out using Topspin Version 2.0 Software.

This article is protected by copyright. All rights reserved. 
For the Meta II decay studies, the samples were illuminated with a $150 \mathrm{~W}$ fiber optic light (Fiber Lite A-200; Dolan-Jenner, Woburn, MA) equipped with a > $495 \mathrm{~nm}$ long-pass filter for $30 \mathrm{sec}$ by placing the light source $\sim 1 \mathrm{~cm}$ away from the NMR tube or sample cuvette.

$1 \mathrm{D}^{19} \mathrm{~F}$ NMR spectra of bovine rhodopsin reconstituted with 14-fluororetinal were measured in Shigemi NMR tubes using a Bruker Avancelll AV600 spectrometer equipped with a $5 \mathrm{~mm} \mathrm{QCl}$ CryoProbe $(\mathrm{HCNF} / \mathrm{z})$. All ${ }^{19} \mathrm{~F}$ NMR chemical shifts were referenced to $\mathrm{CFCl}_{3}$ as external standard. All spectra were recorded with 4997 complex data points (acquisition time $=1 \mathrm{~s}$ ) and without broadband proton decoupling. Comparison against spectra recorded with broadband proton decoupling showed no differences (data not shown).

Transducin activation $\left[^{35}\right.$ SJGTP $\gamma$ S filter binding assay. Binding of transducin to bovine rhodopsin with and without Ce6 was monitored using a $\left[{ }^{35} \mathrm{~S}\right] \mathrm{GTP} \gamma \mathrm{S}$ filter binding assay previously established (25), with several modifications described below. Transducin $\left(G_{t}\right)$ and urea-washed ROS membranes were prepared from dark adapted bovine retinas (W.A. Lawson and Co.) according to previous protocols $(26,27)$ and were stored at $-80{ }^{\circ} \mathrm{C}$ prior to use. $G_{t}$ activation studies were carried out in the absence and at varied concentrations of $\mathrm{Ce} 6$, which was stored as a $100 \mathrm{mM}$ stock solution in DMSO.

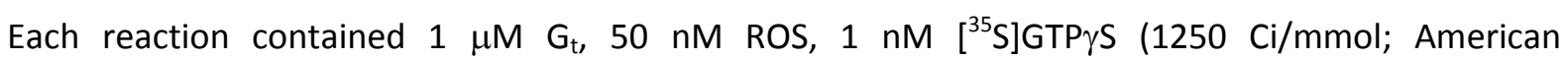
Radiolabeled Chemicals, Inc.), $499 \mathrm{nM}$ cold GTP $\gamma S$ and $1 \mu \mathrm{M}$ GDP. Samples were illuminated with $>495 \mathrm{~nm}$ light for two minutes, then shaken at $250 \mathrm{rpm}$ until the reactions were stopped by filtering $25 \mu \mathrm{l}$ aliquots through nitrocellulose membranes using a Millipore vacuum manifold, washing 3 times with $5 \mathrm{ml}$ of ice-cold wash buffer (10 mM Tris, $\mathrm{pH} 7.4,100 \mathrm{mM} \mathrm{NaCl}, 5 \mathrm{mM} \mathrm{MgCl}, 2 \mathrm{mM}$ DTT). Washed membranes were dried on the manifold then placed in $5 \mathrm{ml}$ of $30 \%$ ScintiSafe LSC-cocktail (Fischer Scientific). Total $\left[{ }^{35}\right.$ S]GTPYS bound was measured using a Beckman LSC 6500 counter. Reactions were performed in triplicates with control samples remaining in the dark.

To determine the relative $\mathrm{IC}_{50}$ values of $\mathrm{Ce} 6$ in $\mathrm{ROS}$ and rhodopsin in DM micelles, $5 \mathrm{nM}$ of either ROS or bovine rhodopsin in DM micelles were incubated with various concentrations of Ce6 for $30 \mathrm{~min}$ post-illumination ( $2 \mathrm{~min}$ ) then filtered as described above. Final DMSO concentrations for each reaction were $1 \%$ and radioligand concentrations were identical to those described above. The $I C_{50}$ values were estimated by fitting the data using the sigmoidal dose-response function of ligand binding provided by Sigmaplot.

This article is protected by copyright. All rights reserved. 
Conservation of residues. The sequence of bovine rhodopsin was submitted to the ConSurf server (39-43), and set to search the "clean Uniprot" database with default settings to collect 150 sequences that sample the list of homologues. The resulting multiple sequence alignment was downloaded and the partial rhodopsin sequence from $M$. niger was added. This was resubmitted to the ConSurf server, and the results plotted in PyMol using the supplied method to colour the PDB 1L9H structure according to conservation score, using the colourblind-friendly key.

The 151 sequence MSA was imported into $R$, as well as the ConSurf output. $R$ scripts were written so that plots could be made indicating the percentage identity to the most common residue for specific positions, and how specific sequences compared with the consensus at these positions. The mean percentage of sequences identical to the consensus was plotted as a dotted line. A table of the query sequences is displayed beneath the plot of percentage identity and colour coded to indicate which sequences are in agreement. The positions identified as Ce6 binding from (13), see red letters in Figure 1, were compared between B. taurus, M. niger, and A. tittmanni.

Statistical Analysis. Data are expressed as mean \pm SD unless otherwise indicated. Differences were evaluated for statistical significance by student t-test and considered statistically significant when $\mathrm{p}<0.05$.

\section{RESULTS}

Absorbance spectroscopy confirms red light sensitivity enhancement but shows no shift in maximal wavelength or dark noise.

First, we investigated the effect of binding of $\mathrm{Ce} 6$ to bovine rhodopsin on the absorbance spectrum of $\mathrm{Ce} 6$ (Figure 2b). The spectrum of $\mathrm{Ce} 6$ changes with addition of rhodopsin, indicating complex formation (Figure 2c). Specifically, there are bathochromic shifts of Ce6's Soret band from $404 \mathrm{~nm}$ to $409 \mathrm{~nm}$ and the $655 \mathrm{~nm}$ peak to $667 \mathrm{~nm}$. Both shifts are known to be associated with aggregation and acidification $(38)(19,20)$, and have been observed as a result of complex formation of Ce6 with polyvinylpyrrolidone $(38)$ and quantum dots $(19,20)$. Thus, Ce6 solutions in the absence of rhodopsin are monomeric under the conditions used, allowing the observation of complex formation through spectral shifting.

This article is protected by copyright. All rights reserved. 
Next, we investigated if the binding of Ce6 results in a red shift of the absorbance maximum of bovine rhodopsin. This is a difficult experiment because of the large background of the Ce6 absorbance spectrum (Figure 2b), its bleaching characteristics when illuminated (data not shown), and its overlap with the $500 \mathrm{~nm}$ absorbance peak of rhodopsin (Figure 3a(I)). We devised a subtraction protocol (see Methods) to alleviate these challenges and obtain difference spectra representing only those absorbance changes relating to bovine rhodopsin (Figure 3a(II)). The absorbance maximum of bovine rhodopsin remains unshifted at $500 \mathrm{~nm}$ and the presence of $\mathrm{Ce} 6$ does not inhibit the conversion to Meta II, as evidenced by the shift to $380 \mathrm{~nm}$. Meta II is susceptible to protonation, forming the characteristic protonated Schiff base peak at $440 \mathrm{~nm}$.

Recently, a report was published indicating that amino acid substitutions characteristic of red opsin structures are associated with increased dark noise due to a "breathing" of the receptor's extracellular domain (33). Because Ce6 binding enhances sensitivity to red light, we therefore investigated if the binding of $\mathrm{Ce} 6$ leads to increased dark noise. Figure $3 \mathrm{c}$ shows that this is not the case. The rhodopsin chromophore is at least as stable, as measured by absorbance at $500 \mathrm{~nm}$, in the dark in the presence of $\mathrm{Ce} 6$ as it is in its absence.

To confirm that $\mathrm{Ce} 6$ actually enhances bovine rhodopsin's sensitivity to red light as reported in a number of previous studies $(8,10,11,34)$, we applied our Ce6 subtraction protocol to rhodopsin illuminated with red light (see Methods). We were able to confirm that the presence of Ce6 enhances bleaching rates not only in retina extracts, but also in our purified system containing only bovine rhodopsin, dodecyl maltoside and Ce6 (Figure 3). The rate enhancement showed dose dependence, with a maximum of 4-fold enhancement (Figure $3 b$ ), consistent with previously reported values (11).

\section{Fluorescence spectroscopy studies show weak binding of Ce6 to bovine rhodopsin.}

There are five tryptophan residues in bovine rhodopsin (Figure 1, blue circles), the fluorescence of which is quenched in the dark by bound retinal and increases upon light activation due to the retinal leaving the protein (1). We reasoned that tryptophan fluorescence may be sensitive to Ce6 binding, either through direct interaction with the tryptophan residues or through conformational effects and/or interference with retinal-protein interactions. Thus, it may serve as a qualitative (or semi-

This article is protected by copyright. All rights reserved. 
quantitative) indicator for binding. Selected tryptophan fluorescence traces of rhodopsin in the dark and upon light-activation in the presence of increasing quantities of $\mathrm{Ce} 6$ are shown in Figure 4a. Tryptophan fluorescence of dark-state rhodopsin remains steady, but is significantly $(p<0.05)$ quenched in the presence of $\mathrm{Ce} 6$, indicating binding of Ce6 to dark-state rhodopsin. At 15.8-fold excess $\mathrm{Ce} 6$ to rhodopsin, the signal is close to the background fluorescence of $\mathrm{Ce} 6$ alone. The arrow indicates light activation, upon which the maximal fluorescence signal obtained after Meta II decay is quenched in a $\mathrm{Ce} 6$ concentration-dependent fashion. This shows that light-activated and subsequent opsin species also bind Ce6. The Meta II-decay half-lives show a small but statistically significant $\left(p=6.2 \times 10^{-3}\right)$ decrease (Figure $\left.4 b\right)$. The differences in fluorescence in the dark and in the light were used to calculate the fractions of free and bound conformations, allowing initial estimations of binding affinities by quantifying the mid-points in the Ce6 concentration dependency curves. This is shown in Figure 4c for the dark (solid circles) and Meta II (open circles) states. The mid-points of the transition are at $0.82 \pm 0.06 \mu \mathrm{M} \mathrm{Ce} 6$ for the Meta II state, and at $2.6 \pm 0.2 \mu \mathrm{M} \mathrm{Ce} 6$ for the dark state.

To assess the selectivity of the bovine rhodopsin binding pocket for Ce6, we compared tryptophan fluorescence quenching by Ce6 with that by chlorophyll a (Chl-a) in Figure 4d. Chl-a is more hydrophobic than $\mathrm{Ce} 6$, which would result in stronger non-specific interactions with rhodopsin, as Chl-a is more readily recruited into the detergent micelles. The results confirm that Ce6 binding is selective, as Chl-a results in a much lower degree of fluorescence quenching, even lower than that observed for Ce6 binding to dark-adapted rhodopsin. Note that Chl-a does have an effect on Meta II half-lives (Figure 4b). This is not unexpected considering the Meta II half-life is highly dependent on its lipid and detergent environment (35-37).

Selective excitation ${ }^{1} \mathrm{H}$ N M R for studying Ce6 binding to bovine rhodopsin.

To complement the fluorescence based binding studies with another, more quantitative method, we used ${ }^{1} \mathrm{H}$ NMR spectroscopy. Due to the large background signals from the detergent peaks present, we used selective excitation (see Methods) in the region downfield of the water and major detergent peaks (Figure $5 b, c$ ). The analysis of selective excitation ${ }^{1} \mathrm{H}$ NMR spectra was as follows. Shown in Figure $5 \mathrm{~b}$ is the selective excitation ${ }^{1} \mathrm{H}$ NMR spectra recorded in the range $7.3-8.7 \mathrm{ppm}$ for $50 \mu \mathrm{M}$ rhodopsin in the absence and presence of Ce6. The peaks observed were sequentially numbered from 1-10 for convenience in each case. Comparison of the spectra in the dark and upon light-activation in the absence of $\mathrm{Ce} 6$ showed an overall decrease in the peak intensities (Figure $5 b(I))$. A signal at peak position 1 appears upon light-activation increases in intensity in a timedependent manner. The intensity of the peak at position 6 increases in a time dependent manner

This article is protected by copyright. All rights reserved. 
after light-activation (Figure $5 b(I)$, black trace with others). Additionally, the peak at position 9 decreases in intensity after 0.5 and 1 hours after light activation (Figure $5 b(I)$, red and blue traces) and disappears completely in the 3 hour post illumination spectra (Figure $5 \mathrm{~b}(\mathrm{I})$, magenta trace). Thus, the time dependent changes observed at peak positions 6 and 9 correspond to the changes associated with structural transition from dark to opsin state. Further, the peaks at positions 2, 3, 7, 9, and 10 originated from the C-terminus residues of rhodopsin (Figure $5 \mathrm{~b}(\mathrm{IV})$ ), indicated as ${ }^{\prime * \prime}$ ). This is because the signals in selective excitation ${ }^{1} \mathrm{H}$ NMR spectra come from flexible residues in the proteins, most likely from the cytoplasmic residues, as this domain is the most flexible as compared to all the other domains in rhodopsin.

In the dark, titrating $10 \mu \mathrm{M}, 50 \mu \mathrm{M}, 400 \mu \mathrm{M}$ and $1 \mathrm{mM}$ of Ce6 to $50 \mu \mathrm{M}$ rhodopsin resulted in changes at peak positions 9 and 10. Shown in Figure $5 \mathrm{c}$ is the overlay of the chemical shift region of rhodopsin for these two peaks in the absence and in the presence of various concentrations of Ce6. It is possible that, at the higher concentrations, there could have been some self-aggregation of Ce6. However, this effect is expected to be moderate at $\mathrm{pH} 6$ (38), and the effects on rhodopsin appear to be progressive from the lower concentrations. The peaks at position 9 and 10 gradually shifted upfield and downfield, respectively (Figure 5c). Except for this change, we did not observe any changes in the other positions for dark state rhodopsin.

The selective excitation ${ }^{1} \mathrm{H}$ NMR spectra of $\mathrm{Ce} 6$ in the absence and presence of various concentrations of rhodopsin in the chemical shift range $8.9 \mathrm{ppm}$ to $10 \mathrm{ppm}$ is shown in Figure $5 \mathrm{a}$. The ${ }^{1} \mathrm{H}$ NMR spectra of $50 \mu \mathrm{M} \mathrm{Ce} 6$ in the absence of rhodopsin gave rise to three chemical shift signals at 9.72, 9.58 and $9.00 \mathrm{ppm}$ (Figure 5a: labeled peaks 1, 2, and 3, respectively). The ${ }^{1} \mathrm{H}$ NMR spectrum of Ce6 acquired in the dark in the presence of $10 \mu \mathrm{M}, 25 \mu \mathrm{M}$ and $50 \mu \mathrm{M}$ rhodopsin showed clear signals from ligand peaks (Figure 5a). The intensity of each signal was less as compared to that observed in the NMR spectrum of $50 \mu \mathrm{M} \mathrm{Ce} 6$ alone (Figure 5a, black trace), indicating restriction in mobility of the ligand resonances in a rhodopsin concentration-dependent manner. Thus, the decrease in signal intensity suggests binding of Ce6 to dark-state rhodopsin. The dark-state rhodopsin $\mathrm{K}_{\mathrm{D}}$ values estimated based on changes in ligand peaks 1,2 and 3 were $16 \pm 7 \mu \mathrm{M}, 16 \pm 9 \mu \mathrm{M}$ and $25 \pm 3 \mu \mathrm{M}$, respectively (Figure $5 \mathrm{~d}(\mathrm{I})$ ), supporting the conclusion from fluorescence spectroscopy that binding of Ce6 to rhodopsin is relatively weak.

We also acquired selective excitation ${ }^{1} \mathrm{H}$ NMR spectra immediately after light-activation to monitor the binding of Ce6 to Meta II (Figure 5e, panel I). Even at the lowest concentration (10 $\mu \mathrm{M})$ of rhodopsin tested, light activation of rhodopsin lead to complete disappearance of Ce6 ligand peak signals, clearly suggesting that the $\mathrm{Ce} 6$ binds to the Meta II state of rhodopsin with much higher affinity than that observed for the dark state. While it was not possible to estimate the $\mathrm{K}_{\mathrm{D}}$ values of

This article is protected by copyright. All rights reserved. 
Ce6 interaction with Meta II state, it is clear that Ce6 binds to Meta II and with higher affinity than observed for dark state. Since Ce6 alone was measured in the presence of the same buffer including detergent, the effects are rhodopsin specific and not merely due to an interaction of Ce6 with the detergent micelles.

Finally, the comparison of the ${ }^{1} \mathrm{H}$ NMR spectra acquired in the absence and presence of rhodopsin $30 \mathrm{~min}$ after light-activation, measuring the binding of $\mathrm{Ce} 6$ to retinal-free opsin, gave rise to detectable ligand peak resonances. Similar to dark state rhodopsin, a decrease in the peak intensities upon addition of rhodopsin protein as compared to free ligand peak intensities were used to estimate an affinity for the opsin-Ce6 complex of $8 \pm 2,11 \pm 2$ and $6 \pm 6 \mu \mathrm{M}$, based on ligand peak 1 , 2 and 3, respectively (Figure 5d(I)).

These results clearly suggest that Ce6 binds to dark, activated Meta II and opsin states of rhodopsin, albeit with different affinities. Ce6 binds quantitatively better to opsin than to dark-state rhodopsin. Furthermore, although it was not possible to estimate the affinity for the interaction between Ce6 and Meta II, it appears to be higher than those for the dark and even the opsin states. Thus, the ${ }^{1} \mathrm{H}$ selective excitation results are consistent with our results obtained from fluorescence spectroscopy, which also indicated that binding of Ce6 to Meta II and to opsin are stronger than to the dark state.

\section{${ }^{19} \mathrm{~F}$ NMR evidence for Ce6 binding and conformational effects on bovine rhodopsin.}

Previous studies are consistent with a cytoplasmic location of the Ce6 binding site (13). To further corroborate the cytoplasmic ligand binding site and study the effects of Ce6 binding on rhodopsin structure and dynamics, we recorded ${ }^{19}$ F NMR spectra of dark- and light-adapted rhodopsin in the absence and presence of Ce6. To enable ${ }^{19} \mathrm{~F}$ NMR, we labeled the protein with a trifluoroethylthiogroup at cysteines Cys-140 and Cys-316 (Figure 1, green circles) in the cytoplasmic domain as described (16). The locations of these label sites are in immediate vicinity to the predicted Ce6 binding site (Table 2 and (13)) and are highlighted in Figure 1 with red letters. The predicted binding site residues are close to the ${ }^{19} \mathrm{~F}$ label attachment sites Cys-140 and Cys-316, demonstrating why these sites are excellent reporters for Ce6 binding. For example, predicted contact residue Lys-141 is the immediate neighbor of Cys-140, and Cys-316 is close to predicted contact residues Gln-312 and Lys66. The resulting ${ }^{19} \mathrm{~F}$ NMR spectra are shown in Figure 6. As observed previously (16), in the absence of $\mathrm{Ce} 6$, there are two distinct peaks at $-65.0 \mathrm{ppm}$ and $-65.4 \mathrm{ppm}$ in the dark (Figure 6a). Upon light activation, these two peaks shifted to their known (16) Meta II positions (Figure 6b, red trace). Meta II peaks decayed to form opsin and gave rise to a broad peak with a maximum at $-65.80 \mathrm{ppm}$ (Figure 6d). The Meta II peaks decreased with a concomitant increase of the opsin peak with an estimated

This article is protected by copyright. All rights reserved. 
half-life of 12.2 minutes for the Cys-140 and 15.7 minutes for the Cys-316 peak. These half-lives fit well with the Meta II decay of rhodopsin determined using fluorescence spectroscopy (Figure 7a).

In the presence of Ce6, detectable shifts of 0.2 ppm were observed in both Cys-140 and Cys316 peaks in the dark (Figure 6a, dotted line), supporting the conclusion that Ce6 binds to the cytoplasmic domain of rhodopsin in the dark. Even more dramatic changes were observed upon lightactivation: the peaks did not shift to the positions of Meta II peaks observed in the absence of Ce6 (Figure 6c). Instead, the peaks shifted directly to a broad peak that does not allow distinguishing Cys140 from Cys-316 at -65.9 ppm suggesting the presence of more than one conformation being formed in the presence of Ce6 in Meta II. This chemical shift is similar, but not identical to the one of the opsin peak in the absence of Ce6, which is observed at $-65.8 \mathrm{ppm}$. The bulk of the peak (with the exception of a small fraction that may represent unbound Meta II or a structure similar to it) did not shift throughout the decay time of opsin supporting the conclusion that the structures formed immediately after light activation are not affected by retinal leaving (Figure 6e). We conclude from these ${ }^{19} \mathrm{~F}$ NMR studies that Ce6 binds at and strongly affects the local environment of both cysteines in the cytoplasmic face of rhodopsin, in the dark and in the light. Because of the similarity of the ${ }^{19} \mathrm{~F}$ chemical shift in the presence of Ce6 after light activation to opsin, we measured the UV/Visible absorption spectrum of rhodopsin. Acidification allows distinguishing between Meta II and free retinal absorbances, because only Meta II shifts to $440 \mathrm{~nm}$ as a result of Schiff base protonation. We observed a $440 \mathrm{~nm}$ peak after addition of $1 \% \mathrm{HCl}(\mathrm{v} / \mathrm{v})$ in the presence of Ce6 immediately after illumination, but not 1.5 hours after illumination, indicating retinal is still bound to Lys-296 in the first spectrum but absent in the later opsin state (data not shown) (39). This clearly suggests that the ${ }^{19}$ F NMR peak at -65.9 ppm observed immediately after illumination in the presence of Ce6 arises from an altered (opsin resembling) Meta state, rather than immediate formation of retinal-free opsin. This state then decays to opsin by releasing retinal as in the absence of Ce6 with a half-life similar to unbound Meta II (Figure 4b).

Finally, the presence of Ce6 also affects another previously observed phenomenon (16), that of loss of ${ }^{19} \mathrm{~F}$ label over long periods of time (hours), seen by the appearance of a sharp peak at $69 \mathrm{ppm}$, corresponding to the free label (Figure 6f). If we assume that the formation of free ${ }^{19} \mathrm{~F}$ label is due to nucleophilic attack of the trifluoroethyl-cysteine disulfide bond by amino acids in the protein, this suggests that Ce6 remains bound to rhodopsin even after Meta II decay, presumably preventing the motions required for such an attack (Figure $6 f$, Figure $7 b, c$ ).

This article is protected by copyright. All rights reserved. 
Reconstitution of bovine rhodopsin with fluorinated retinal followed by ${ }^{19} \mathrm{~F}$ NMR spectroscopy. The lack of changes in retinal absorbance characteristics make it unlikely that $\mathrm{Ce} 6$ binds close to the retinal binding pocket as would have been required for a photosynthesis-like energy transfer mechanism proposed previously (11). To more quantitatively test the potential effect (or lack thereof) of $\mathrm{Ce} 6$ on the local structural environment experienced by the retinal, we synthesized fluorinated retinal and reconstituted it into rhodopsin (see Methods). The ${ }^{19} \mathrm{~F}$ NMR spectra of rhodopsin carrying the fluorinated retinal in the presence and absence of $\mathrm{Ce} 6$ are shown in Figure $8 \mathrm{~b}$. The position of the broad retinal peak at $-123.76 \mathrm{ppm}$ (referenced externally to $\mathrm{CFCl}_{3}$ ) is in agreement with published values for rhodopsin bound 9-cis-14-fluororetinal (40), and is essentially unaffected by $\mathrm{Ce} 6$ binding, supporting the conclusion that Ce6 most likely does not bind near the retinal.

\section{Conformational changes in the extracellular and transmembrane domains induced by $\mathrm{Ce} 6$ binding} at the cytoplasmic domain of bovine rhodopsin.

Since Ce6 appears to bind to the cytoplasmic domain and not near the retinal, the red light sensitization mechanism is likely mediated by an allosteric mechanism, as proposed earlier (12). To directly monitor such allosteric effects, we measured ${ }^{1} \mathrm{H}^{15} \mathrm{~N}$ heteronuclear single quantum correlation (HSQC) NMR spectra of $\alpha-\varepsilon^{-15} \mathrm{~N}$-labeled tryptophan rhodopsin at $37^{\circ} \mathrm{C}$. Out of the five tryptophans present in rhodopsin, Trp-35 is in the extracellular domain, and the remaining four tryptophans are distributed among different transmembrane helices (Figure 1). An overlay of the conventional HSQC spectra of $\alpha-\varepsilon^{-{ }^{15}} \mathrm{~N}$-tryptophan labeled rhodopsin in the absence and presence of a 10 -fold excess of $\mathrm{Ce} 6$ is shown in Figure 9 . Based on recent studies $(6,41)$, the signals observed at $7.2,7.7,8.2,8.3$, and $8.7 \mathrm{ppm}$ in the backbone region and $11.4,10.6,10.1,10.2$, and $11.1 \mathrm{ppm}$ in the side chain region were assigned to Trp-161, Trp-35, Trp-265, Trp-175 and Trp-126, respectively (Figure 9).

In the presence of $\mathrm{Ce} 6$, the overall spectrum looked similar to rhodopsin alone, except for the following changes: Three out of the five indole side chain signals were shifted in the presence of Ce6 as compared to rhodopsin alone (Figure 9, labeled in blue). A shift of $+0.11,+0.06$ and $-0.03 p p m$ for the peaks corresponding to Trp-161, Trp-126 and Trp-35 in the side chain regions were observed. In the backbone region, peaks corresponding to Trp-126 and Trp-161 in the presence of Ce6 were shifted by +0.07 and $+0.11 \mathrm{ppm}$. Further, an increase in the intensity of the Trp-161 peak was also observed in the backbone region, suggesting that Trp-161 might be experiencing increased flexibility

This article is protected by copyright. All rights reserved. 
in the presence of $\mathrm{Ce} 6$. These studies clearly suggest that binding of $\mathrm{Ce} 6$ in the cytoplasmic domain induces changes in the transmembrane region, in particular Trp-126 on helix 3 and Trp-161 on helix 4 located near the 11-cis retinal ligand binding pocket, but towards the cytoplasmic domain (Figure 9). Trp-265 on helix 6, which packs directly against the retinal, shows very little changes in both the backbone and sidechain regions. This is consistent with the lack of changes in rhodopsin-bound fluororetinal in the ${ }^{19}$ F NMR spectra recorded in the absence vs. in the presence of Ce6 (Figure $8 \mathrm{~b}(\mathrm{II}))$, hence further confirming that $\mathrm{Ce} 6$ does not bind near the retinal and its binding to the cytoplasmic domain does not lead to perturbation of the retinal binding pocket.

\section{Ce6 interferes with $G_{t}$ binding to bovine rhodopsin.}

The functional role of Meta II rhodopsin is to activate $G_{t}$ and initiate signaling. The observed differences in ${ }^{19} \mathrm{~F}$ NMR spectra in the presence and absence of $\mathrm{Ce} 6$ as well as the docking results suggest that Ce6 may interfere with $\mathrm{G}_{\mathrm{t}}$ activation. To test this hypothesis we measured the activation of $\mathrm{G}_{\mathrm{t}}$ by light-activated rhodopsin using a $\left[{ }^{35} \mathrm{~S}\right] \mathrm{GTP} \gamma \mathrm{S}$ filter binding assay in the absence and presence of Ce6. $G_{t}$ activation was inhibited in a concentration-dependent manner in the presence of Ce6 (Figure 10c). $\mathrm{IC}_{50}$ values in the range of $65-150 \mathrm{nM}$ were obtained for $5 \mathrm{nM}$ rhodopsin and $1 \mu \mathrm{M} \mathrm{G}$ in DM micelles and ROS membranes (Figure 10a). A time course of light-dependent activation of $\mathrm{G}_{\mathrm{t}}$ by rhodopsin in bovine ROS was measured in the presence and absence of a 25 -fold excess Ce6 (Figure 10b). At 120 seconds post-illumination a sample free of Ce6 displayed near saturation in activation, whereas an identical sample containing 25-fold excess Ce6 displayed no activation as compared to dark controls (Figure 10b). These studies suggest that Ce6 interferes with $\mathrm{G}_{\mathrm{t}}$ binding to rhodopsin in both ROS and DM micelles.

Further support for an interference of $\mathrm{Ce} 6$ with $G_{t}$ binding came from tryptophan fluorescence studies with a high affinity analog of the $G_{t}$ C-terminal peptide (VLEDLKSCGLF) $(42,43)$. In the presence of both a 5 -fold excess Ce6 and 1500 -fold excess $G_{t}$, peptide fluorescence quenching is greater than in the presence of either compound alone (Figure 10d), consistent with a competition of $\mathrm{Ce} 6$ with the $\mathrm{G}_{\mathrm{t}}$ peptide for an overlapping binding site.

This article is protected by copyright. All rights reserved. 


\section{Sequence conservation in bovine and deep-sea cytoplasmic Ce6 binding sites}

Because we carried out all our experiments with bovine, rather than M. niger rhodopsin, we checked if the cytoplasmic Ce6 binding site is also present in deep-sea dragonfish. Figure 11 shows that the binding site is conserved, validating bovine rhodopsin as a good model system for $M$. niger rhodopsin. A multiple sequence alignment was assembled of rhodopsins from 150 species and compared with $M$. niger rhodopsin. The percentage of species identical with B. taurus, M. niger, and A. tittmanni at the positions of the $\mathrm{Ce} 6$ binding site are indicated by the heights of the bars in figure 11a. For 18 of the 20 positions for which the partial sequence of $M$. niger has a recorded value all three sequences are in agreement with one another and the most common residue at that position. Of the 27 positions identified as the Ce6 binding site, 19 have a higher percentage identity than the average of all positions (shown as a dotted line in Figure 11a). This high level of concentration is better shown through the output of ConSurf analysis in Figure 11b. This shows the Ce6 (and G protein) binding site is highly conserved in general and between bovine and dragonfish rhodopsins.

\section{DISCUSSION}

This paper makes the following major contributions: (1) We conducted a comprehensive literature survey and critical analysis of the evidence relating to red light sensitivity in deep sea ocean fish. (2) We show conclusively that Ce6 is a small molecule ligand for rhodopsin. (3) The affinity of bovine rhodopsin for Ce6 is weak, in the $\mu \mathrm{M}$ range, and is higher for Meta II and opsin states than for the dark state. (4) Ce6 does not cause a shift in absorbance maximum to the red, nor appears to strongly alter the structure of the retinal binding pocket. (5) Ce6 does alter the cytoplasmic domain structure in dark and light-activated states. (6) Ce6 exerts global effects on bovine rhodopsin structure and dynamics in line with our previous finding of modulation of conserved signaling pathways. (7) Ce6 binding inhibits $\mathrm{G}$ protein activation.

The affinity calculated based on fluorescence and NMR spectroscopies differ slightly from each other with dark-state rhodopsin $K_{D}$ values estimated by NMR of $16 \pm 7 \mu \mathrm{M}$ to $25 \pm 3 \mu \mathrm{M}$ (depending on the Ce6 peak used), as compared to $2.6 \pm 0.2 \mu \mathrm{M}$ based on fluorescence spectroscopy. One possible reason could be the differences in concentration of $\mathrm{Ce} 6$ and rhodopsin needed for fluorescence and NMR spectroscopy, respectively. In particular, higher concentrations of Ce6 up to 1 $\mathrm{mM}$ were used in ${ }^{1} \mathrm{H}$ NMR experiments, in contrast to the absorbance and fluorescence experiments in which the concentrations were in the micromolar range. It is known that Ce6 aggregates at high concentrations and acidic conditions $(19,20)(38)$ and we can therefore not rule out that the NMR

This article is protected by copyright. All rights reserved. 
experiments were conducted with at least partially aggregated Ce6 (these aggregates are not detectable by eye, since the solutions were clear). It is thus possible that aggregation may be the reason for the slight discrepancy between the affinities obtained from NMR versus fluorescence experiments, based on the different concentration regimes necessary for these experiments.

The discovery that begs the most discussion is that $\mathrm{Ce} 6$ would enhance red light sensitivity in deep-sea ocean dragonfish but inhibit $G$ protein activation. Although we carried out all our experiments with bovine rhodopsin, the high sequence conservation between dragonfish and bovine rhodopsin indicates that our results are extendable to dragonfish. We suspect the presence of $\mathrm{Ce} 6$ and related compounds in the dragonfish retina is simply a consequence of diet, without a specific role in vision. The latter hypothesis is supported by recent localization studies that show these compounds to be distributed throughout all retinal layers in varying amounts, including the inner retina and the retinal plexiform layers (44). Lipid-soluble compounds such as Ce6 may be enriched in the plexiform layers due to their higher density membranes (44). Given that in darkness, even the human eye is able to detect dim red light of wavelengths $>650 \mathrm{~nm}$ (and even longer wavelengths at higher light intensities), there is no need to invoke Ce6 binding as a requirement for rhodopsin activation. Instead, chlorophyll based small molecules may universally increase red light activation of rhodopsin but also inhibit the propagation of the signal (should they compete for similar binding sites as $\mathrm{G}$ proteins).

Ce6 binds at the "heart of the action," where the largest conformational changes are observed after light-activation $(45,46)$, and where critical and highly conserved residues are exposed to interact with $\mathrm{G}_{\mathrm{t}}(45,46)$. For example, Glu-134 and Arg-135 are the residues of the D/ERY motif, which almost $100 \%$ conserved in Class A GPCRs (47). We therefore suspect that Ce6 does not only bind to rhodopsin but also to other GPCRs, likely affecting processes other than vision (also see (13) for further discussion of the generality of allosteric modulation in the GPCR family). An energy transfer mechanism for the enhancement of activation by triplet sensitization as detailed in (11) remains a possibility, though the large distance between the cytoplasmic Ce6 binding site and transmembrane almost extracellular retinal location eliminates the efficient use of energy transfer mechanisms. An alternative mechanism has been proposed recently based on the allosteric adjustment of the retinal binding by Ce6 (13). It is interesting to note that as can be seen in Table 2, A1 and A2 chromophore have both been observed for $M$. niger, with $\lambda$ max of $\sim 522$ and $\sim 548 \mathrm{~nm}$, respectively. This could conceivably result in more efficient activation of rhodopsin by red light, and even more so if the mechanism of activation is by energy transfer from Ce6.

This article is protected by copyright. All rights reserved. 
Ce6 and the G protein are not the only ligands that can bind in the cytoplasmic domain. Independent predictions of small molecule binding pockets for rhodopsin based on analysis of high connectivity of residues in proteins (48), identified the CP domain as a likely source for binding sites, in addition to the known retinal pocket. Nine residues of the CP site are located within $5 \AA$ of the $G_{t}$ peptide and Ce6 binding sites (Figure 1, red letters; Table 2). Indeed, a number of additional small molecules capable of binding to bovine rhodopsin and modulating $G_{t}$ activation have been identified (49), although no further conformational studies have been conducted to check for putative allosteric effects. Thus, while the physiological relevance of Ce6 binding for red light detection may be limited, its study has unveiled a novel allosteric modulator, that may prove general for the GPCR family.

\section{Author contributions}

All studies were designed and conducted under the supervision and guidance of Judith KleinSeetharaman. James Mitchell conducted all absorbance spectroscopy experiments, the ConSurf analysis and prepared fluorinated retinal reconstitution NMR samples. Naveena Yanamala conducted fluorescence binding and Meta II decay assays, ${ }^{1} \mathrm{H}$ NMR based binding assays, ${ }^{19} \mathrm{~F}$ NMR of bovine rhodopsin with cytoplasmic labels, ${ }^{1} \mathrm{H},{ }^{15} \mathrm{~N}-\mathrm{HSQC}$ spectroscopy of tryptophan labeled samples. Yi Lei Tan under the supervision of Daniel Nietlispach conducted the ${ }^{19} \mathrm{~F}$ NMR experiments with fluorinated retinal and bovine rhodopsin samples. Eric $E$. Gardner performed $G_{t}$ activation studies. Kalyan Tirupula conducted the fluorescence studies involving the peptide. M Sheves synthesized the fluororetinal. All authors approved the manuscript.

\section{Acknowledgements}

This work was in part supported by an International Incoming Fellowship of the Seventh Framework Programme (FP7) Marie Curie Actions (to JKS), a fellowship by the DAAD-Helmholtz Society to FB, the Sofya Kovalevskaya Prize of the Humboldt Foundation, Germany / Zukunftsinvestitionsprogramm der Bundesregierung Deutschland, National Science Foundation grants EIA0225636 and CAREER CC044917 to JKS. We would like to thank Dr. Inja Byeon (Structural Biology, University of Pittsburgh) for help with the set-up of selective excitation NMR experiments.

This article is protected by copyright. All rights reserved. 


\section{References:}

1. Farrens, D. L., and Khorana, H. G. (1995) Structure and Function in Rhodopsin Measurement of the rate of metarhodopsin II decay by fluorescence spectroscopy. J. Biol. Chem. 270, 50735076

2. Heck, M., Schädel, S. A., Maretzki, D., Bartl, F. J., Ritter, E., Palczewski, K., and Hofmann, K. P. (2003) Signaling states of rhodopsin. Formation of the storage form, metarhodopsin III, from active metarhodopsin II. J. Biol. Chem. 278, 3162-3169

3. Heck, M., Schädel, S. A., Maretzki, D., and Hofmann, K. P. (2003) Secondary binding sites of retinoids in opsin: characterization and role in regeneration. Vision Res. 43, 3003-3010

4. Vogel, R., Siebert, F., Mathias, G., Tavan, P., Fan, G., and Sheves, M. (2003) Deactivation of Rhodopsin in the Transition from the Signaling State Meta II to Meta III Involves a Thermal Isomerization of the Retinal Chromophore CN Double Bond. Biochemistry. 42, 9863-9874

5. Vogel, R., Siebert, F., Zhang, X.-Y., Fan, G., and Sheves, M. (2004) Formation of Meta III during the Decay of Activated Rhodopsin Proceeds via Meta I and Not via Meta II. Biochemistry. 43, 9457-9466

6. Stehle, J., Silvers, R., Werner, K., Chatterjee, D., Gande, S., Scholz, F., Dutta, A., Wachtveitl, J., Klein-Seetharaman, J., and Schwalbe, H. (2014) Characterization of the Simultaneous Decay Kinetics of Metarhodopsin States II and III in Rhodopsin by Solution-State NMR Spectroscopy. Angew. Chem. 126, 2110-2116

7. Douglas, R. H., Partridge, J. C., Dulai, K., Hunt, D., Mullineaux, C. W., Tauber, A. Y., and Hynninen, P. H. (1998) Dragon fish see using chlorophyll. Nature. 393, 423-424

8. Isayama, T., Alexeev, D., Makino, C. L., Washington, I., Nakanishi, K., and Turro, N. J. (2006) An accessory chromophore in red vision. Nature. 443, 649

9. Douglas, R. H., Partridge, J. C., Dulai, K. S., Hunt, D. M., Mullineaux, C. W., and Hynninen, P. H. (1999) Enhanced retinal longwave sensitivity using a chlorophyll-derived photosensitiser in Malacosteus niger, a deep-sea dragon fish with far red bioluminescence. Vision Res. 39, 28172832

10. Washington, I., Zhou, J., Jockusch, S., Turro, N. J., Nakanishi, K., and Sparrow, J. R. (2007) Chlorophyll derivatives as visual pigments for super vision in the red. Photochem. Photobiol. Sci. 6, 775-779

11. Ilyas Washington, Celeste Brooks, Nicholas J. Turro, * and, and Nakanishi*, K. (2004) Porphyrins As Photosensitizers To Enhance Night Vision. JACS 126, 9892-9893.

12. Balem, F., Yanamala, N., and Klein-Seetharaman, J. (2009) Additive Effects of Chlorin E6 and Metal Ion Binding on the Thermal Stability of Rhodopsin In Vitrot. Photochem. Photobiol. 85, 471-478

This article is protected by copyright. All rights reserved. 
13. Woods, K. N., Pfeffer, J., and Klein-Seetharaman, J. (2017) Chlorophyll-Derivative Modulation of Rhodopsin Signaling Properties through Evolutionarily Conserved Interaction Pathways. Front. Mol. Biosci. 10.3389/fmolb.2017.00085

14. Needleman, S. B., and Wunsch, C. D. (1970) A general method applicable to the search for similarities in the amino acid sequence of two proteins. J. Mol. Biol. 48, 443-453

15. Henikoff, S., and Henikoff, J. G. (1992) Amino acid substitution matrices from protein blocks. Proc. NatI. Acad. Sci. U. S. A. 89, 10915-10919

16. Klein-Seetharaman, J., Getmanova, E. V., Loewen, M. C., Reeves, P. J., and Khorana, H. G. (1999) NMR spectroscopy in studies of light-induced structural changes in mammalian rhodopsin: Applicability of solution 19F NMR. Proc. Natl. Acad. Sci. 96, 13744-13749

17. Steinberg, G., Ottolenghi, M., and Sheves, M. (1993) pKa of the protonated Schiff base of bovine rhodopsin. A study with artificial pigments. Biophys. J. 64, 1499-1502

18. Oprian, D. D., Molday, R. S., Kaufman, R. J., and Khorana, H. G. (1987) Expression of a synthetic bovine rhodopsin gene in monkey kidney cells. Proc. Natl. Acad. Sci. U. S. A. 84, 8874-8878

19. Rotomskis, R., Valanciunaite, J., Skripka, A., Steponkiene, S., Spogis, G., Bagdonas, S., and Streckyte, G. (2013) Complexes of functionalized quantum dots and chlorin e6 in photodynamic therapy. Lith J Phys 53, 57-68.

20. Kundelev, E. V., Orlova, A. O., Maslov, V. G., Baranov, A. V., and Fedorov, A. V. (2017) Circular dichroism spectroscopy of complexes based on semiconductor quantum dots and chlorin e6 molecules. Opt. Eng. 56, 047102

21. Birdsall, B., King, R. W., Wheeler, M. R., Lewis, C. A., Jr., Goode, S. R., Dunlap, R. B., and Roberts, G. C. (1983) Correction for light absorption in fluorescence studies of protein-ligand interactions. Anal Biochem. 132, 353-61

22. Parker, C. A. (1968) Photoluminescence of Solutions: With Applications to Photochemistry and Analytical Chemistry, Elsevier Publishing Company

23. Hwang, T. L., and Shaka, A. J. (1995) Water Suppression That Works. Excitation Sculpting Using Arbitrary Wave-Forms and Pulsed-Field Gradients. J. Magn. Reson. A. 112, 275-279

24. Stott, K., Stonehouse, J., Keeler, J., Hwang, T.-L., and Shaka, A. J. (1995) Excitation Sculpting in High-Resolution Nuclear Magnetic Resonance Spectroscopy: Application to Selective NOE Experiments. J. Am. Chem. Soc. 117, 4199-4200

25. Wessling-Resnick, M., and Johnson, G. L. (1987) Transducin interactions with rhodopsin. Evidence for positive cooperative behavior. J. Biol. Chem. 262, 12444-12447

26. Baehr, W., and Morita, E. A. Characterization of Bovine Rod Outer Segment G-protein

27. Papermaster, D. S. (1982) [35] Preparation of antibodies to rhodopsin and the large protein of rod outer segments. in Methods in Enzymology, pp. 240-246, Biomembranes - Part H: Visual Pigments and Purple Membranes - I, Academic Press, 81, 240-246

This article is protected by copyright. All rights reserved. 
28. Glaser, F., Pupko, T., Paz, I., Bell, R. E., Bechor-Shental, D., Martz, E., and Ben-Tal, N. (2003) ConSurf: Identification of Functional Regions in Proteins by Surface-Mapping of Phylogenetic Information. Bioinformatics. 19, 163-164

29. Landau, M., Mayrose, I., Rosenberg, Y., Glaser, F., Martz, E., Pupko, T., and Ben-Tal, N. (2005) ConSurf 2005: the projection of evolutionary conservation scores of residues on protein structures. Nucleic Acids Res. 33, W299-W302

30. Ashkenazy, H., Erez, E., Martz, E., Pupko, T., and Ben-Tal, N. (2010) ConSurf 2010: calculating evolutionary conservation in sequence and structure of proteins and nucleic acids. Nucleic Acids Res. 38, W529-W533

31. Celniker, G., Nimrod, G., Ashkenazy, H., Glaser, F., Martz, E., Mayrose, I., Pupko, T., and BenTal, N. (2013) ConSurf: Using Evolutionary Data to Raise Testable Hypotheses about Protein Function. Isr. J. Chem. 53, 199-206

32. Ashkenazy, H., Abadi, S., Martz, E., Chay, O., Mayrose, I., Pupko, T., and Ben-Tal, N. (2016) ConSurf 2016: an improved methodology to estimate and visualize evolutionary conservation in macromolecules. Nucleic Acids Res. 44, W344-W350

33. Yue, W. W. S., Frederiksen, R., Ren, X., Luo, D.-G., Yamashita, T., Shichida, Y., Cornwall, M. C., and Yau, K.-W. (2017) Spontaneous activation of visual pigments in relation to openness/closedness of chromophore-binding pocket. eLife. 6, e18492

34. Degl'Innocenti, A., Rossi, L., Salvetti, A., Marino, A., Meloni, G., Mazzolai, B., and Ciofani, G. (2017) Chlorophyll derivatives enhance invertebrate red-light and ultraviolet phototaxis. Sci. Rep. 10.1038/s41598-017-03247-1

35. Mitchell, D. C., Straume, M., and Litman, B. J. (1992) Role of sn-1-saturated,sn-2polyunsaturated phospholipids in control of membrane receptor conformational equilibrium: effects of cholesterol and acyl chain unsaturation on the metarhodopsin I .tautm. metarhodopsin II equilibrium. Biochemistry. 31, 662-670

36. Brown, M. F. (1994) Modulation of rhodopsin function by properties of the membrane bilayer. Chem. Phys. Lipids. 73, 159-180

37. Szundi, I., Lewis, J. W., and Kliger, D. S. (2007) Effect of Digitonin on the Rhodopsin Meta IMeta II Equilibrium ๆ. Photochem. Photobiol. 81, 866-873

38. Paul, S., Selvam, S., Heng, P. W. S., and Chan, L. W. (2013) Elucidation of Monomerization Effect of PVP on Chlorin e6 Aggregates by Spectroscopic, Chemometric, Thermodynamic and Molecular Simulation Studies. J. Fluoresc. 23, 1065-1076

39. Sakamoto, T., and Khorana, H. G. (1995) Structure and function in rhodopsin: the fate of opsin formed upon the decay of light-activated metarhodopsin II in vitro. Proc. Natl. Acad. Sci. 92, 249-253

40. Leticia U. Colmenares, Walter P. Niemczura, Alfred E. Asato, and, and Liu*, R. S. H. (1996) A 19F NMR Study of Rhodopsin Analogs: Use of Vinylfluororetinal Chromophores

This article is protected by copyright. All rights reserved. 
41. Werner, K., Lehner, I., Dhiman, H. K., Richter, C., Glaubitz, C., Schwalbe, H., Klein-Seetharaman, J., and Khorana, H. G. (2007) Combined solid state and solution NMR studies of $\alpha, \varepsilon-15 \mathrm{~N}$ labeled bovine rhodopsin. J. Biomol. NMR. 37, 303-312

42. Kisselev, O. G., Meyer, C. K., Heck, M., Ernst, O. P., and Hofmann, K. P. (1999) Signal transfer from rhodopsin to the G-protein: Evidence for a two-site sequential fit mechanism. Proc. Natl. Acad. Sci. 96, 4898-4903

43. Arimoto, R., Kisselev, O. G., Makara, G. M., and Marshall, G. R. (2001) Rhodopsin-Transducin Interface: Studies with Conformationally Constrained Peptides. Biophys. J. 81, 3285-3293

44. Douglas, R. H., Genner, M. J., Hudson, A. G., Partridge, J. C., and Wagner, H.-J. (2016) Localisation and origin of the bacteriochlorophyll-derived photosensitizer in the retina of the deep-sea dragon fish Malacosteus niger. Sci. Rep. 6, 39395

45. Oldham, W. M., and Hamm, H. E. (2008) Heterotrimeric G protein activation by G-proteincoupled receptors. Nat. Rev. Mol. Cell Biol. 9, 60-71

46. Janz, J. M., and Farrens, D. L. (2004) Rhodopsin Activation Exposes a Key Hydrophobic Binding Site for the Transducin $\alpha$-Subunit C Terminus. J. Biol. Chem. 279, 29767-29773

47. Sakmar, T. P. (2002) Structure of rhodopsin and the superfamily of seven-helical receptors: the same and not the same. Curr. Opin. Cell Biol. 14, 189-195

48. Illingworth, C. J. R., Scott, P. D., Parkes, K. E. B., Snell, C. R., Campbell, M. P., and Reynolds, C. A. Connectivity and binding-site recognition: Applications relevant to drug design. J. Comput. Chem. 31, 2677-2688

49. Taylor, C. M., Barda, Y., Kisselev, O. G., and Marshall, G. R. (2008) Modulating G-Protein Coupled Receptor/G-Protein Signal Transduction by Small Molecules Suggested by Virtual Screening. J. Med. Chem. 51, 5297-5303

50. Judith Klein-Seetharaman, §, John Hwa, §, Kewen Cai, §, Christian Altenbach, , Wayne L. Hubbell, and, and H. Gobind Khorana*, $\S(1999)$ Single-Cysteine Substitution Mutants at Amino Acid Positions 55-75, the Sequence Connecting the Cytoplasmic Ends of Helices I and II in Rhodopsin: Reactivity of the Sulfhydryl Groups and Their Derivatives Identifies a Tertiary Structure that Changes upon Light-Activation ${ }^{\dagger} \neq$

51. Bowmaker, J. K., Dartnall, H. J. A., and Herring, P. J. (1988) Longwave-sensitive visual pigments in some deep-sea fishes: segregation of 'paired' rhodopsins and porphyropsins. J. Comp. Physiol. A. 163, 685-698

52. Crescitelli, F. (1990) Adaptations of visual pigments to the photic environment of the deep sea. J. Exp. Zool. 256, 66-75

53. O'Day, W. T., and Fernandez, H. R. (1974) Aristostomias scintillans (Malacosteidae): a deep-sea fish with visual pigments apparently adapted to its own bioluminescence. Vis. Res. 14, 545-50

54. Partridge, J. C., and Douglas, R. H. (1995) Far-red sensitivity of dragon fish. Nature. 375, 21-22

This article is protected by copyright. All rights reserved. 
55. Douglas, R. H., Partridge, J. C., and Marshall, N. J. (1998) The eyes of deep-sea fish I: Lens pigmentation, tapeta and visual pigments. Prog. Retin. Eye Res. 17, 597-636

56. Knowles, A., and Dartnall, H. J. A. (1977) The Photobiology of Vision, Academic Press

57. Partridge, J. C., Shand, J., Archer, S. N., Lythgoe, J. N., and Groningen-Luyben, W. A. H. M. van (1989) Interspecific variation in the visual pigments of deep-sea fishes. J. Comp. Physiol. A. 164, 513-529

58. Somiya, H. (1982) 'Yellow Lens' Eyes of a Stomiatoid Deep-Sea Fish, Malacosteus niger. Proc. $R$. Soc. Lond. B Biol. Sci. 215, 481-489

59. Denton, E. J., Gilpin-Brown, J. B., and Wright, P. G. (1970) On the "filters" in the photophores of mesopelagic fish and on a fish emitting red light and especially sensitive to red light. J Physiol. 208, 72P-73P

\section{Figure Captions}

Figure 1: Secondary structure model of bovine rhodopsin highlighting the positions of key amino acids discussed in this manuscript. The attachment site of retinal, Lys296 in helix VII, is shown as a blue circle with white letter K. Of the ten cysteines in rhodopsin, only Cys-140 and Cys-316 are circled and labeled in green because they are the only ones accessible to chemical derivatization in the dark. All five tryptophan residues in rhodopsin are labeled and circled in blue. The disulfide bond between Cys-110 and Cys-187 is represented by a dotted line. The $5 \AA$ predicted Ce 6 binding pocket residues in rhodopsin are highlighted in bold and colored in red (also see Table 2).

Figure 2: Characteristics of Ce6. (a) The chemical structure of Ce6. (b) Normalized absorbance spectrum of Ce6. The spectrum shows the Soret band at $402 \mathrm{~nm}$, and the Q-band, comprising four peaks in the range $450-700 \mathrm{~nm}$. Also shown is a spectrum of Ce6 in the presence of rhodopsin. In this spectrum there are bathochromic shifts of the Soret band to $407 \mathrm{~nm}$ and the $655 \mathrm{~nm}$ peak in the Qband to $665 \mathrm{~nm}$, both known to be associated with aggregation and acidification (38). These shifts indicate complex formation between bovine rhodopsin and $\mathrm{Ce} 6$, analogous to the complex formation of $\mathrm{Ce} 6$ with polyvinylpyrrolidone $(38)$ and quantum dots $(19,20)$.

This article is protected by copyright. All rights reserved. 
Figure 3: Absorbance spectroscopy. (a) Absorbance spectra of Rhodopsin bleaching by $630 \mathrm{~nm}$ light in the presence of Ce6. (I): Raw spectra with $\mathrm{Ce} 6$ added to half the concentration of bovine rhodopsin. The initial rhodopsin spectrum was multiplied by the dilution factor from adding $\mathrm{Ce} 6$ and subtracted from the spectrum with $\mathrm{Ce} 6$ added to obtain a spectrum for the $\mathrm{Ce} 6$ contribution (see Methods). Between each subsequent spectrum, the sample was illuminated through a $630 \mathrm{~nm}$ longpass filter for 30 seconds. A spectrum was taken after exposure to the room's ambient light to ensure all bleaching was caused by the light source.

(II): Subtraction of the $\mathrm{Ce} 6$ contribution from the bleaching timecourse. The $\mathrm{Ce} 6$ contribution was calculated by multiplying the reference spectrum acquired by subtraction by the ratio between the $A_{665}$ of the reference and that timepoint. The dashed line running through $500 \mathrm{~nm}$ is shown to indicate that without the $\mathrm{Ce} 6$ contribution the rhodopsin spectrum is not altered. The black line shows the initial spectrum. The green line in a(I) shows the first spectrum with $\mathrm{Ce} 6$ added. Spectra at 60, 120 and 300 seconds are shown as blue, red, and purple lines respectively. (b) Enhancement of bleaching rates by Ce6. Remaining rhodopsin was estimated using the $A_{500}$ of Ce6 subtracted spectra. These were normalised between the initial spectrum and the $495 \mathrm{~nm}$ bleached spectrum to calculate the extent of bleaching, and single exponential decay curves were fitted to the time points to estimate rate constants. Ce6 concentration was estimated using the $\mathrm{A}_{664}$ of the initial $\mathrm{Ce} 6$ spectrum, and rhodopsin from the range of $A_{500}$ values. The dotted line indicates the rate of bleaching without Ce6. (c) Dark noise in the presence of Ce6. Rhodopsin was kept at the temperatures indicated in the presence and absence of $\sim 5 \times \mathrm{Ce} 6$. Ce6's contribution was subtracted as described, and the dark noise estimated using the $A_{500}$ at each time point. Similar results to $37^{\circ} \mathrm{C}$ were observed at $25^{\circ} \mathrm{C}$, but omitted for clarity.

Figure 4: Evidence for weak binding from fluorescence spectroscopy. (a) Fluorescence emission traces were obtained at $330 \mathrm{~nm}$ (excitation wavelength $=280 \mathrm{~nm}$ ) in the presence of increasing amounts of $\mathrm{Ce} 6$ up to 15.8 times the rhodopsin concentration.

(b) Meta-II decay rates of bovine rhodopsin in the absence and presence of Ce6. The half-lives of Meta-Il decay of bovine rhodopsin in the absence and presence of additives (chlorophyll-a (open circles) and Ce6 (filled circles)) at various concentrations indicated. All samples contained a rhodopsin concentration of $0.5 \mu \mathrm{M}$. The half-lives of Meta-II decay of rhodopsin in the presence of Ce6 correspond to mean \pm SD from three independent experiments.

This article is protected by copyright. All rights reserved. 
(c) Quantification of binding constants. Plots of the relative populations of bound and free states in the dark (filled circles) and in the light (open circles) using the data shown in (a). Mean \pm SD from three independent experiments performed on separate days from different stocks of Rhodopsin samples.

(d) Effects of illuminated Ce6 on dark and light activated bovine rhodopsin. Fluorescence traces of rhodopsin in the presence of dark Chlorophyll-a (green circles), illuminated Chlorophyll-a (black circles), dark Ce6 (yellow circles) and illuminated Ce6(red circles). A rhodopsin concentration of 0.5 $\mu \mathrm{M}$ was used to record each fluorescence trace. $A^{(* \prime}$ represents that the sample is illuminated.

Figure 5. Evidence for weak binding from ${ }^{1} H$ NMR spectroscopy. (a) Overlay of the selective excitation ${ }^{1} \mathrm{H}$ NMR spectra of $50 \mu \mathrm{M}$ Ce6 in the absence and presence of $10 \mu \mathrm{M}, 25 \mu \mathrm{M}$ and $50 \mu \mathrm{M}$ bovine rhodopsin in the dark.

(b) Monitoring binding of Ce6 to dark-adapted and light-activated rhodopsin. Overlay of onedimensional selective excitation ${ }^{1} \mathrm{H}$ NMR spectra in the dark and at different time points after lightactivation of (I) rhodopsin (II) rhodopsin with $10 \mu \mathrm{M}$ Ce6 and (III) rhodopsin with $50 \mu \mathrm{M}$ Ce6. (IV) 1 mM C-terminal peptide corresponding to the last nine amino acids of rhodopsin in phosphate buffer and $0.3 \%$ DM. In I, II, and III the NMR traces corresponding to the dark, 0.5, 1 and 3 hours after lightactivation are colored in black, red, blue and magenta, respectively. NMR samples contained $50 \mu \mathrm{M}$ of rhodopsin in $20 \mathrm{mM}$ sodium phosphate buffer ( $\mathrm{pH} 6.0$ ), $0.3 \% \mathrm{DM}$ and $10 \% \mathrm{D}_{2} \mathrm{O}$.

(c) One dimensional selective excitation ${ }^{1} \mathrm{H}$ NMR spectra of dark rhodopsin without and at various concentrations of Ce6. The ${ }^{1} \mathrm{H}$ NMR spectra of dark state rhodopsin in the absence (black trace) and in the presence of $10 \mu \mathrm{M}$ (red trace), $50 \mu \mathrm{M}$ (blue trace), $400 \mu \mathrm{M}$ (green trace), $1 \mathrm{mM}$ Ce6 (magenta trace) in the chemical shift range $7.4 \mathrm{ppm}$ to $7.55 \mathrm{ppm}$ is shown. These peaks correspond to the positions 9 and 10 in Figure 4b.

(d) The binding curve for the ligand peaks in ${ }^{1} \mathrm{H}$ NMR spectra in the dark (I) and 1.5 hours post illumination (II). Peak 1, 2 and 3 are labeled using red circles, blue squares and green diamonds colors respectively.

(e) Change in the selective excitation ${ }^{1} \mathrm{H}$ NMR spectra of Meta II (I) and opsin (II) state of rhodopsin in the absence and at various concentrations of Ce6. For trace colouring refer to $4 \mathrm{c}$ above.

This article is protected by copyright. All rights reserved. 
Figure 6. Conformational consequences of binding at the cytoplasmic surface probed by NMR spectroscopy of ${ }^{19} \mathrm{~F}$-labeled cysteines. Cysteine residues at positions 140 and 316 in rhodopsin were labeled by trifluoroethylthio (TET) groups as described previously (50). A total of $7 \mathrm{mg}$ of rhodopsin in $20 \mathrm{mM}$ sodium phosphate buffer with $9 \% \mathrm{n}$-dodecyl-b-D-maltoside (DM) and $10 \% \mathrm{D}_{2} \mathrm{O}$ at pH 6.0 was used for each sample.

(a) Dark state rhodopsin (labeled with ${ }^{19} \mathrm{~F}$ at positions Cys-140 and Cys-316) without Ce6 (solid line) and with Ce6 (dotted line) at 1:5 rhodopsin to Ce6 ratio.

(b) ${ }^{19}$ F NMR spectra of dark and light activated rhodopsin in the absence of Ce6.

(c) ${ }^{19}$ F NMR spectra of dark and light activated rhodopsin in the presence of Ce6 at 2.5 -fold excess.

(d,e) Time dependent changes in the CP domain of rhodopsin upon light-activation. Overlay of one-dimensional ${ }^{19} \mathrm{~F}$ NMR traces as a function of time in the dark and upon light-activation in the absence (d) and presence (e) of Ce6. (f) Overlay of one-dimensional ${ }^{19} \mathrm{~F}$ NMR spectra of rhodopsin 3 hours after illumination in the absence (blue line) and presence of Ce6 (red line).

Figure 7. (a) Comparison of the decay of Meta II peaks of Cys-140 and Cys-316 measured by NMR spectroscopy with Meta II decay kinetics obtained using fluorescence spectroscopy. Meta II decay of rhodopsin was measured by fluorescence at $10 \%$ DM to mimic the large detergent concentrations obtained as a result of concentrating elution fractions for NMR samples at $0.5 \mathrm{mM}$ rhodopsin concentration. The results obtained with fluorescence is shown as black circles and those from NMR spectroscopy as red and green circles. Peaks 1 and 2 correspond to the Meta II peaks of Cys-140 and Cys-316 peaks after light-activation. The calculated decay rate constants for rhodopsin (fluorescence), peak 1 and peak 2 are $0.0454,0.0566$ and $0.0385 \mathrm{~min}^{-1}$, respectively. A rhodopsin concentration of $0.5 \mu \mathrm{M}$ and $0.5 \mathrm{mM}$ was used to record fluorescence and NMR spectra, respectively. The results obtained from these two independent methods are in good agreement.

(b) Decay of rhodopsin after light-activation. Decay of the broad peak at $-65.8 \mathrm{ppm}$ and at -65.9 ppm for rhodopsin (blue line in 5f) and rhodopsin plus Ce6 (red line in 5f), respectively in the absence (filled circles) and presence (open circles) of 8.5 fold excess Ce6. The decay rate of opsin in the presence of $\mathrm{Ce} 6$ was decreased by a factor of 2 .

This article is protected by copyright. All rights reserved. 
(c) Free label formation in the absence and presence of Ce6. Plot of the formation of the free label peak (its position in the spectrum is indicated in $5 f$ ) in the absence (filled circles) and presence (open circles) of Ce6. Less than $20 \%$ of the ${ }^{19} \mathrm{~F}$ label appeared as free label after 16 hours. Data were fitted using the exponential decay function for (b) and linear function for (c).

Figure 8. (a) Reconstitution of bovine rhodopsin with 9-cis-14-fluororetinal (I): Fraction of bleached rhodopsin reconstituted over time measured by the increase in $A_{523}$ relative to the $A_{500}$ difference between dark adapted and light-activated rhodopsin. At $20^{\circ} \mathrm{C}$ (triangles) yield is $29.3 \%$, at $4{ }^{\circ} \mathrm{C}$ (circles), $42.5 \%$.

(II) Comparison of $A_{523}$ change between samples with (triangles) and without added $\mathrm{Ce} 6$ (circles). Addition of $\mathrm{Ce} 6$ and retinal at the same time complicates spectra too much to compare light activated spectrum, so time points are compared with the 2 minute measurement.

(b) ${ }^{19} \mathrm{~F}$ NMR spectra of bovine rhodopsin reconstituted with fluorinated retinal. $1 \mathrm{D}^{19} \mathrm{~F}$ NMR of 150 $\mu \mathrm{M}$ 9-cis-14-fluororhodopsin in DM, in the absence (magenta; 54318 scans; $r g=912$ ) or presence (blue; 60110 scans; $r g=912$ ) of $\mathrm{Ce} 6$, and $150 \mu \mathrm{M}$ 9-cis-14-fluororetinal in 1.3\% DM in the absence of Ce6 (black; 50176 scans; $r g=256$ ). All spectra were processed with line broadening of $80 \mathrm{~Hz}$. The

${ }^{19} \mathrm{~F}$ chemical shift of rhodopsin-bound 9-cis-14-fluororetinal is $-123.8 \mathrm{ppm}$, with linewidths of 1.9 ppm and $1.8 \mathrm{ppm}$ in the absence and presence of Ce6, respectively. Other peaks correspond to recorded values (40) for free 9-cis retinal with fluorines at positions 10 (-119.6 ppm), 10 and 14 ($128.5 \mathrm{ppm})$, and 14 (-131.7 ppm).

Figure 9. Allosteric effects of Ce6 binding by ${ }^{1} \mathrm{H},{ }^{15} \mathrm{~N}$ NMR spectroscopy. The ${ }^{1} \mathrm{H}$ NMR spectra of ${ }^{15} \mathrm{~N}-$ $\alpha$ - $\varepsilon$-tryptophan labeled bovine rhodopsin in the absence and presence of 10 -fold excess Ce 6 are colored in black and red traces respectively. The chemical shift changes in the backbone and sidechain regions upon addition of $\mathrm{Ce} 6$ are highlighted in bold and labeled in red and blue, respectively.

Figure 10: Concentration-dependent inhibition of $G_{t}$ activation by Ce6. (a) Decrease in $G_{t}$ activation monitored with $5 \mathrm{nM}$ bovine ROS (open circles) and $5 \mathrm{nM}$ purified bovine rhodopsin in DM micelles (closed circles). All reactions were incubated for 30 minutes following illumination (2

This article is protected by copyright. All rights reserved. 
minutes) and stopped by filtering through nitrocellulose membranes. (b) Time course of $\mathbf{G}_{\mathbf{t}}$ activation with illuminated rhodopsin in the absence (filled circles) and presence of a 25 -fold excess of $\mathrm{Ce} 6$ (open circles) over rhodopsin concentration. The rhodopsin concentration used was $0.25 \mu \mathrm{M}$. The data in (a) was fitted with the sigmoidal dose-response non-linear regression function and using the exponential growth function for (b). Data represents mean \pm SD from three independent experiments performed using different stocks of rhodopsin samples.

(c) Modulation of $G_{t} / G_{t}$ peptide binding to rhodopsin in the presence of Ce6. $G_{t}$ activation in ROS by the $\left[{ }^{35} \mathrm{~S}\right] \mathrm{GTP} \gamma \mathrm{S}$ filter binding assay. The relative ratio of light versus dark radioligand counts is plotted against the $\mathrm{Ce} 6$ concentration in $\mathrm{nM}$. $\mathrm{G}_{\mathrm{t}}$ activation is inhibited by the presence of $\mathrm{Ce} 6$. Mean $\pm S D$ from three independent experiments. (d) Binding of $G_{t}$ peptide and Ce6 to rhodopsin in DM micelles using fluorescence spectroscopy. The rhodopsin concentration used was $0.25 \mu \mathrm{M}$. Data in (c) was fitted with the exponential decay function and using the exponential growth function for (d).

Figure 11. Consurf analysis: Interspecies conservation of putative $\mathrm{Ce} 6$ binding residues. (a): The alignment of 150 rhodopsin sequences identified by ConSurf from B. taurus with a partial sequence for $\boldsymbol{M}$. niger rhodopsin. The percentage of sequences identical to query sequences is indicated by colour bars, and identity to the consensus sequence by the grey bar. The dotted line indicates mean identity to the consensus sequence, not including gaps, for the whole alignment: $63.0 \%$. The table below is colour coded by which query sequences are identical with the consensus at that position.

(b) The PDB 1L9H crystal structure of rhodopsin coloured with the ConSurf scores. The Ce6 binding pocket identified by docking is represented as a surface, the rest in cartoon representation.

This article is protected by copyright. All rights reserved. 
Table 1. Overview of previous studies on rhodopsin pigments in deep-sea ocean fishes.

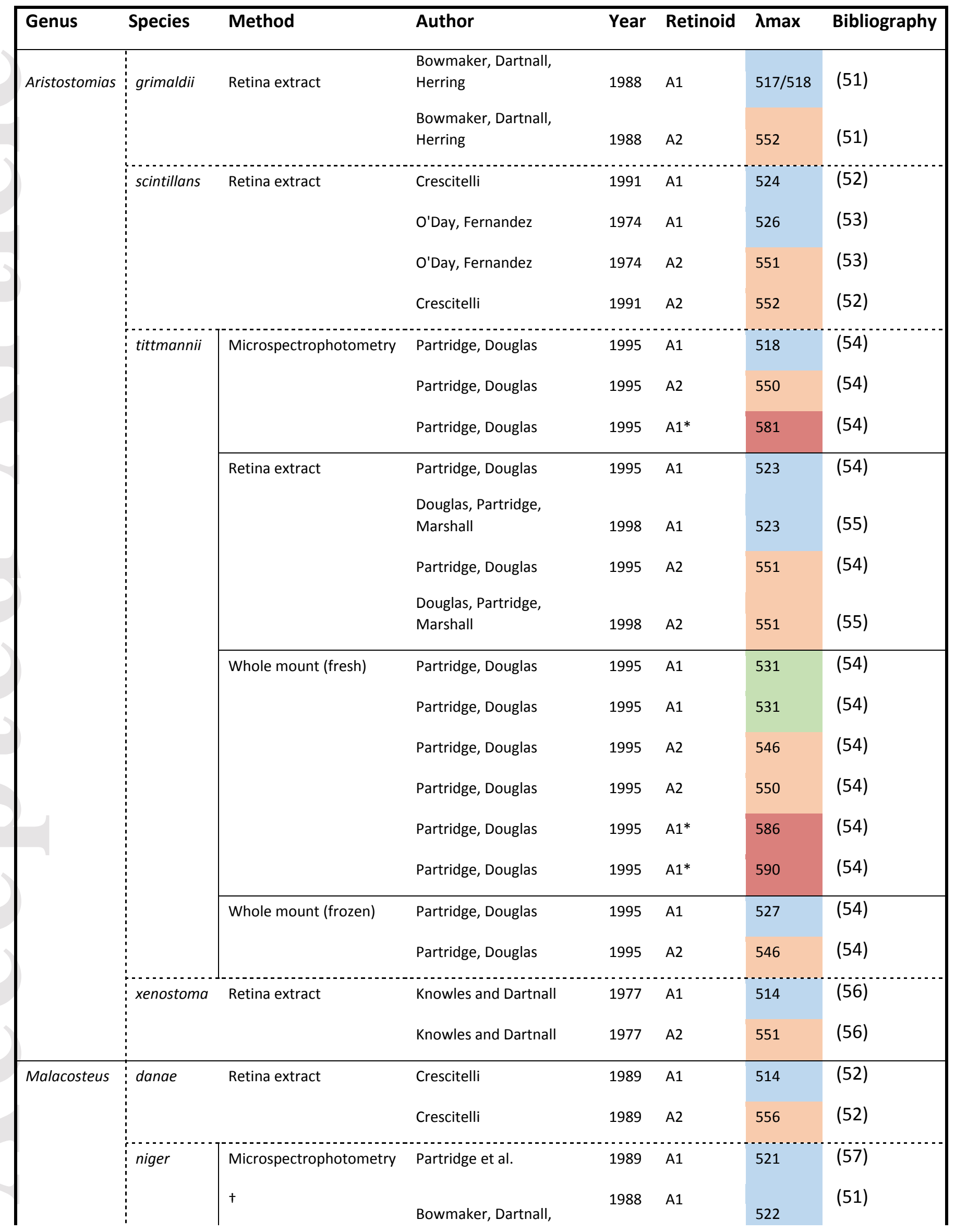

This article is protected by copyright. All rights reserved. 


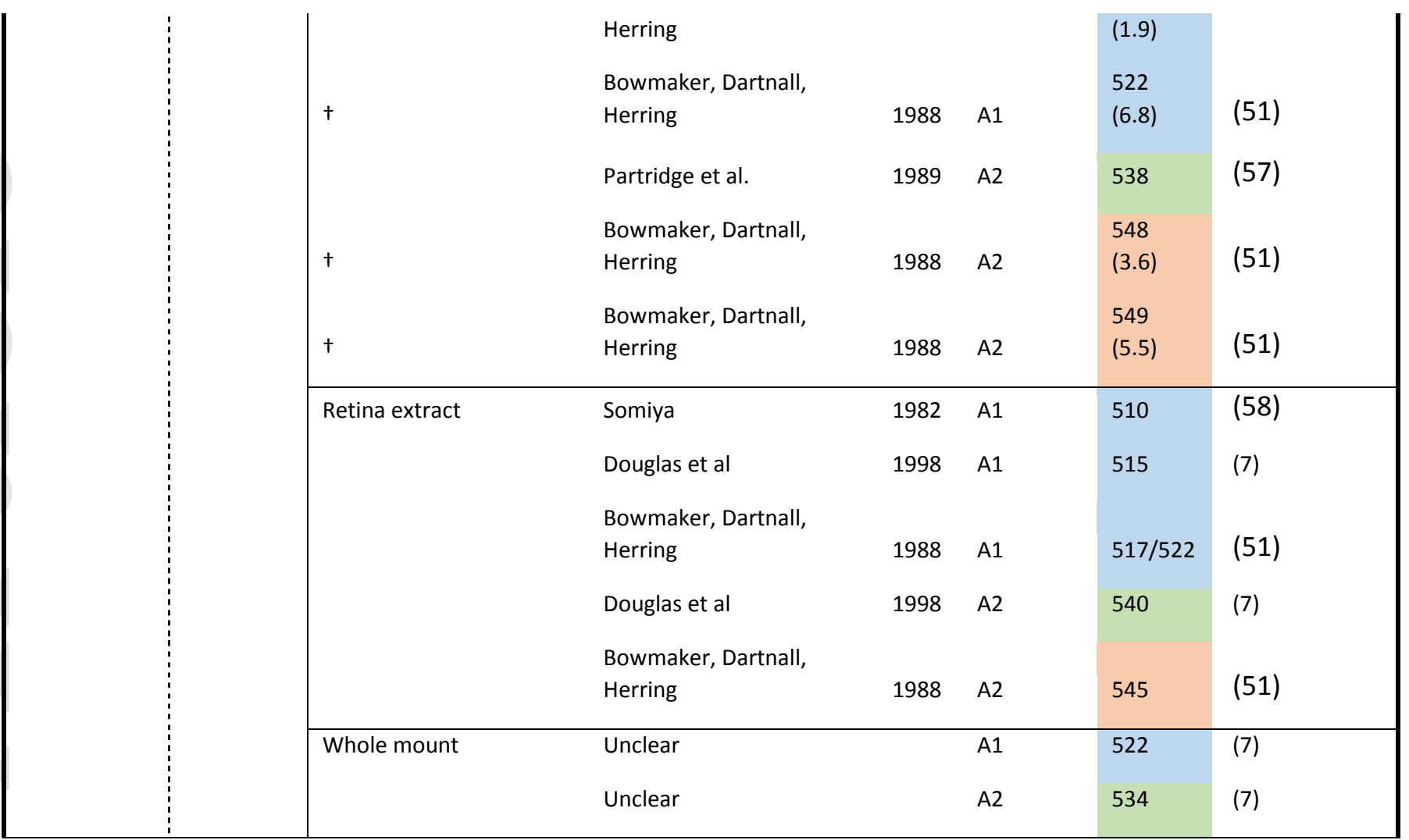

$\lambda_{\max }$ coloured by wavelength: $<530 \mathrm{~nm}$ : blue, 531 to $541 \mathrm{~nm}$ : green, 542 to $563 \mathrm{~nm}$ : orange, $>564$

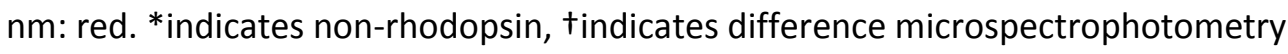


Table 2: Residues identified for $\mathrm{Ce} 6$ binding and $\mathrm{G}$ protein binding sites. $\mathrm{Ce} 6$ binding sites from docking of $\mathrm{Ce} 6$ with rhodopsin in (13).

\begin{tabular}{|c|c|c|c|}
\hline & & $\begin{array}{l}\text { Ce6 } \\
\text { binding }\end{array}$ & $\begin{array}{l}\text { G protein } \\
\text { binding }\end{array}$ \\
\hline K & 66 & & $\checkmark$ \\
\hline $\mathrm{K}$ & 67 & $\checkmark$ & $\checkmark$ \\
\hline L & 68 & $\checkmark$ & \\
\hline $\mathrm{R}$ & 69 & $\checkmark$ & \\
\hline $\mathrm{T}$ & 70 & $\checkmark$ & \\
\hline$P$ & 71 & $\checkmark$ & $\checkmark$ \\
\hline $\mathrm{L}$ & 72 & $\checkmark$ & $\checkmark$ \\
\hline $\mathrm{E}$ & 134 & $\checkmark$ & $\checkmark$ \\
\hline $\mathrm{R}$ & 135 & $\checkmark$ & $\checkmark$ \\
\hline V & 138 & $\checkmark$ & $\checkmark$ \\
\hline V & 139 & & $\checkmark$ \\
\hline C & 140 & & $\checkmark$ \\
\hline K & 141 & $\checkmark$ & $\checkmark$ \\
\hline $\mathrm{P}$ & 142 & & $\checkmark$ \\
\hline$M$ & 143 & & $\checkmark$ \\
\hline S & 144 & & $\checkmark$ \\
\hline$N$ & 145 & & $\checkmark$ \\
\hline$F$ & 146 & & $\checkmark$ \\
\hline$R$ & 147 & $\checkmark$ & $\checkmark$ \\
\hline$F$ & 148 & $\checkmark$ & \\
\hline G & 149 & $\checkmark$ & \\
\hline $\mathrm{E}$ & 150 & $\checkmark$ & $\checkmark$ \\
\hline L & 226 & & $\checkmark$ \\
\hline $\mathrm{T}$ & 229 & & $\checkmark$ \\
\hline V & 230 & & $\checkmark$ \\
\hline
\end{tabular}

This article is protected by copyright. All rights reserved. 


E $232 \checkmark$

A 233

A $234 \checkmark$

Q $236 \sqrt{ }$

Q $237 \checkmark \checkmark$

Q $238 \sqrt{ }$

E $239 \checkmark$

T $243 \checkmark$

A $246 \checkmark$

E $\quad 247 \quad \checkmark$

E $249 \quad \checkmark$

$\mathrm{V} 250 \checkmark \checkmark \checkmark$

M $253 \quad \checkmark$

V $254 \quad \checkmark$

N $310 \checkmark$

F $313 \checkmark$

S $334 \checkmark$

T $335 \checkmark$

T $336 \checkmark$

Q $344 \checkmark$

V $345 \checkmark$

A $346 \checkmark$

P $347 \quad \checkmark$

This article is protected by copyright. All rights reserved. 


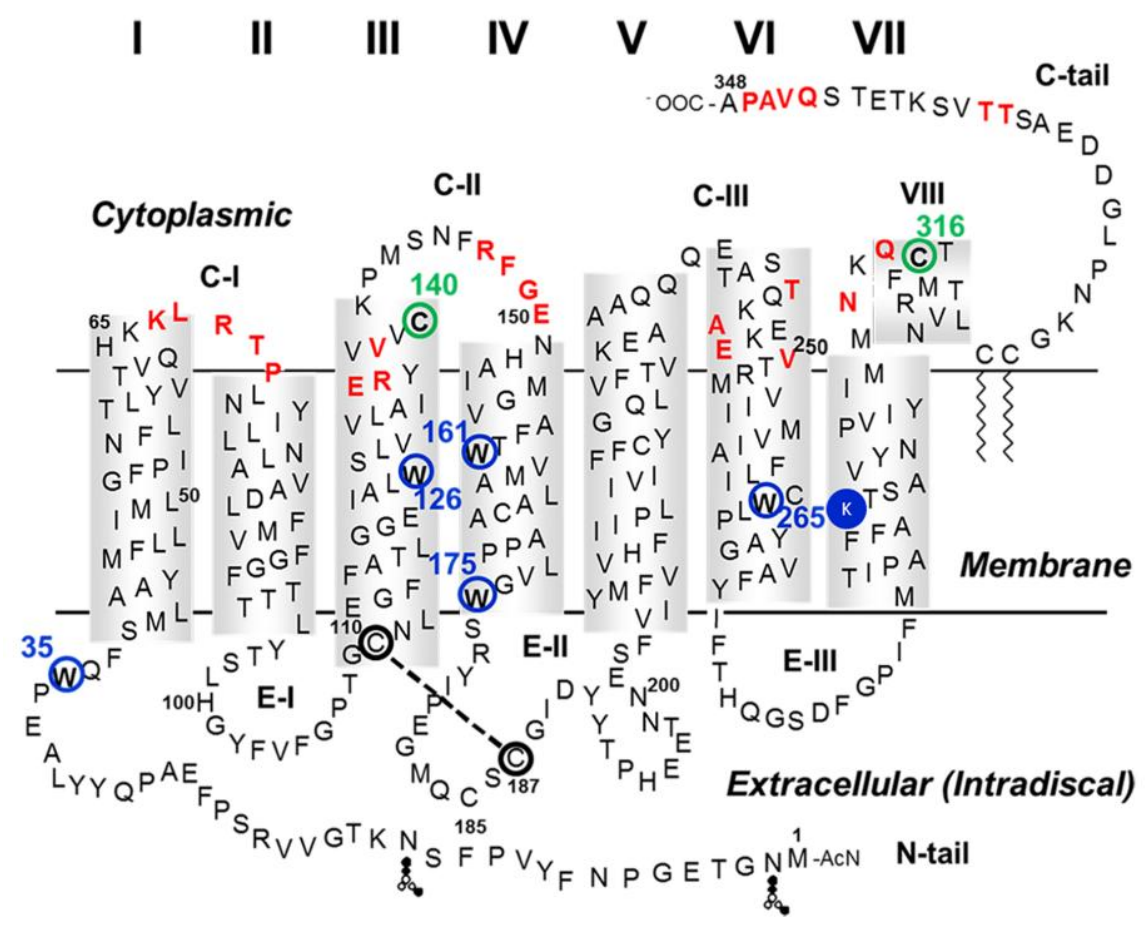

(a)

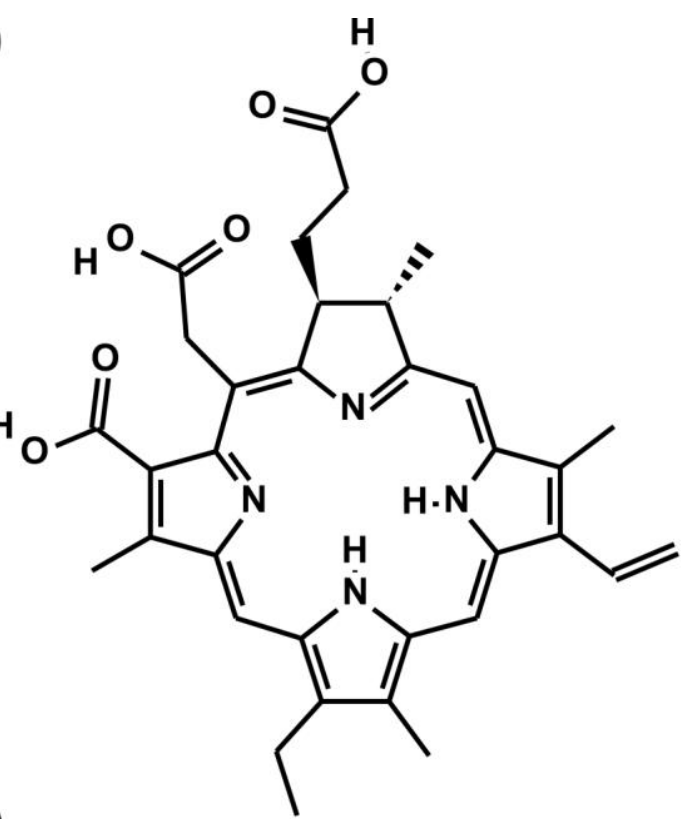

(b)

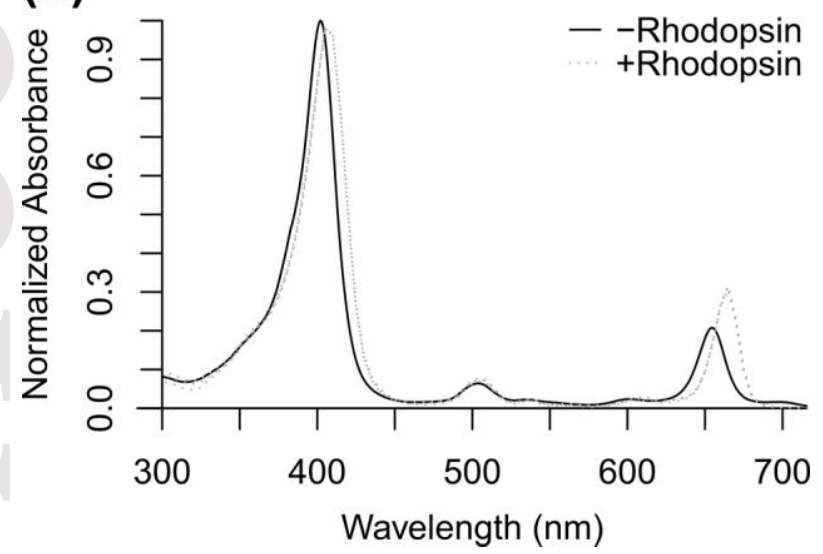

This article is protected by copyright. All rights reserved. 
(a)
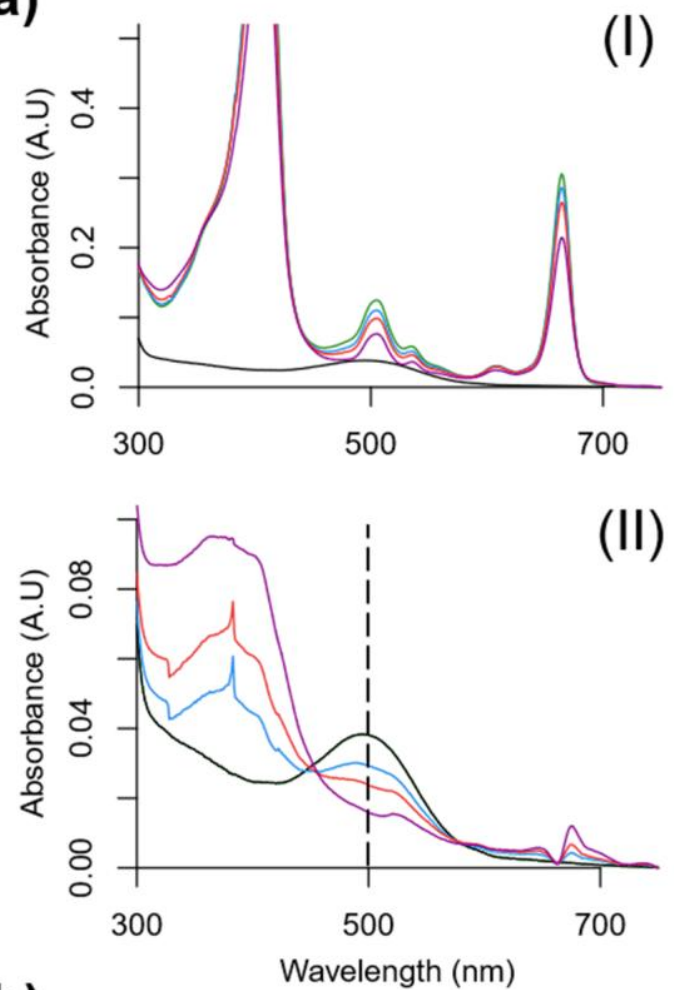

(b)

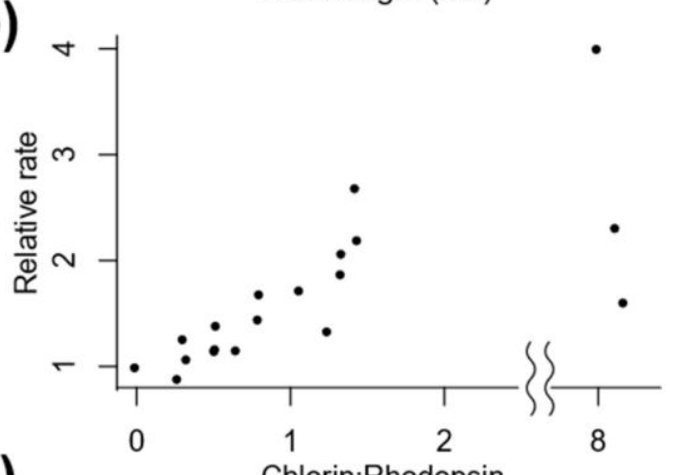

(c)



This article is protected by copyright. All rights reserved. 
(a)
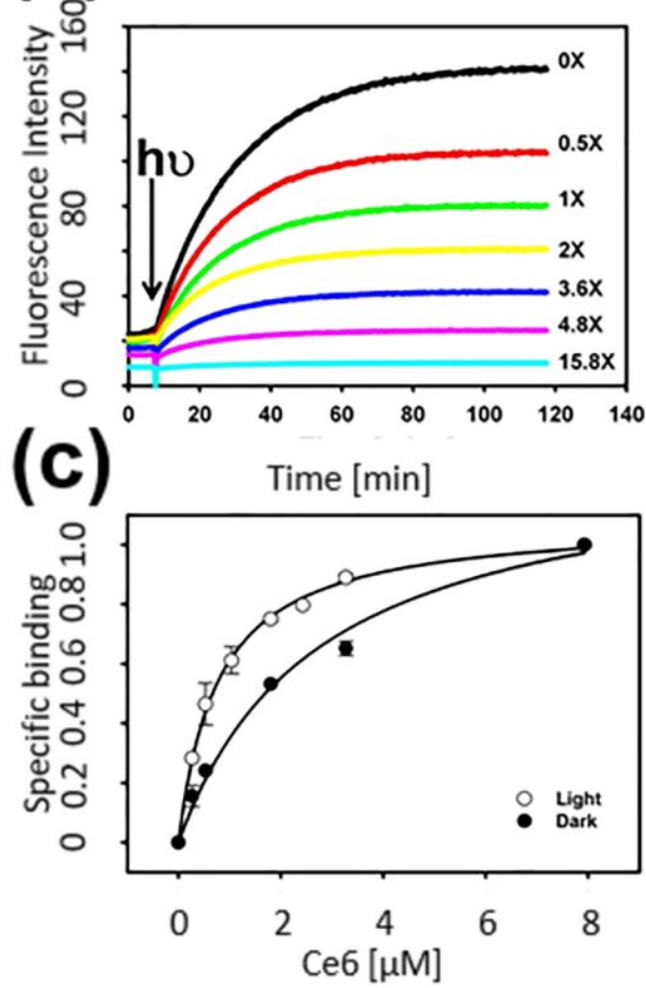

(b)
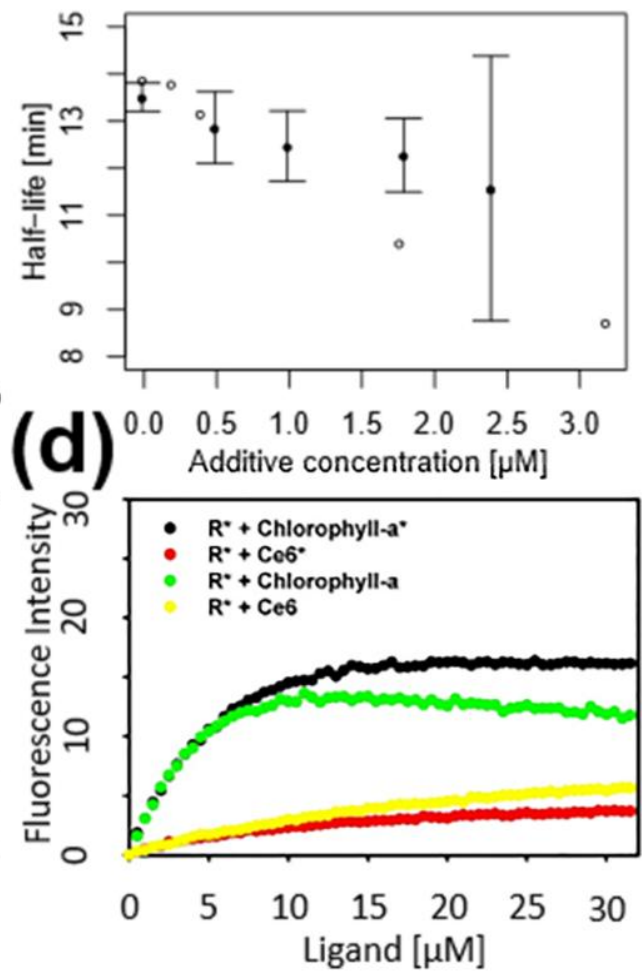

This article is protected by copyright. All rights reserved. 
(a)

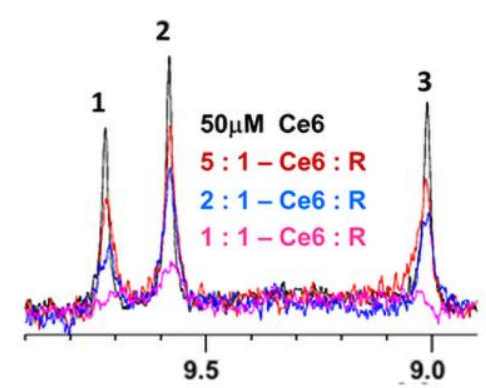

${ }^{1} \mathrm{H}$ Chemical Shift

(c)

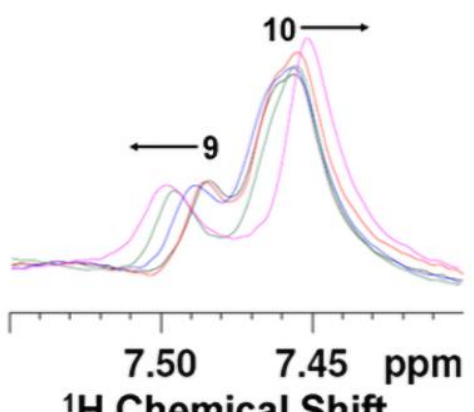

(d) ${ }^{1} \mathrm{H}$ Chemical Shift
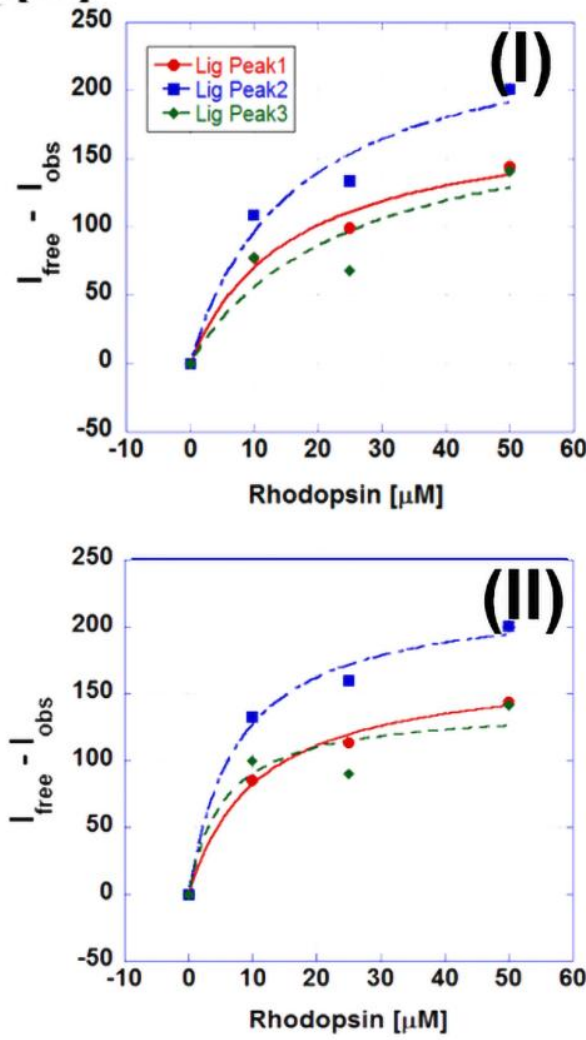

(b)

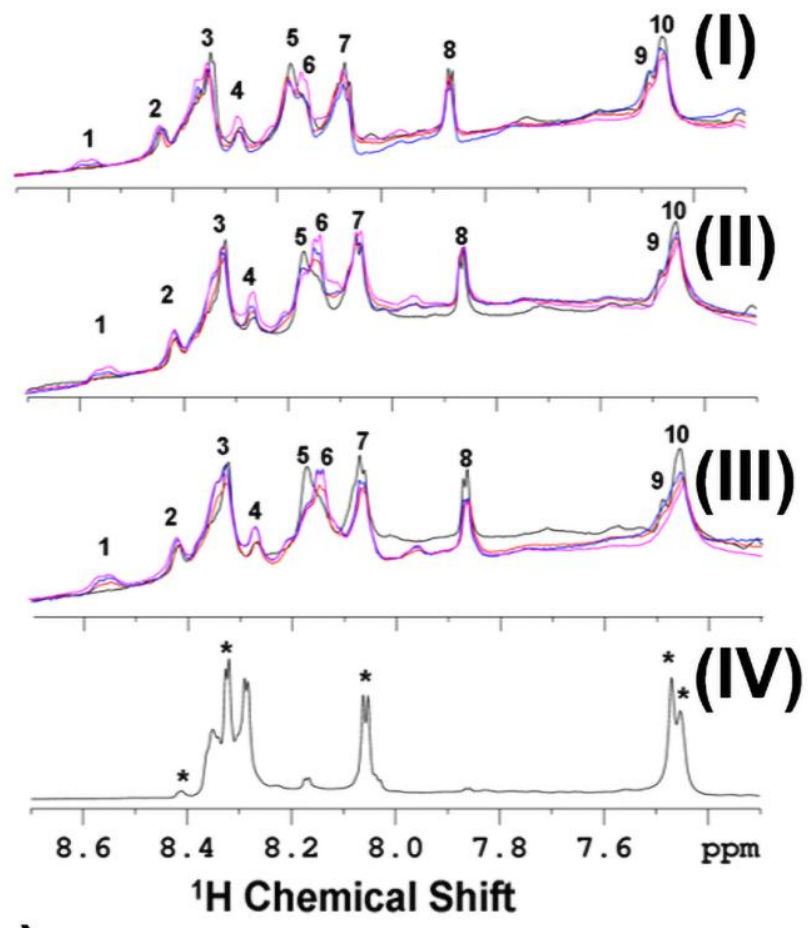

(e)
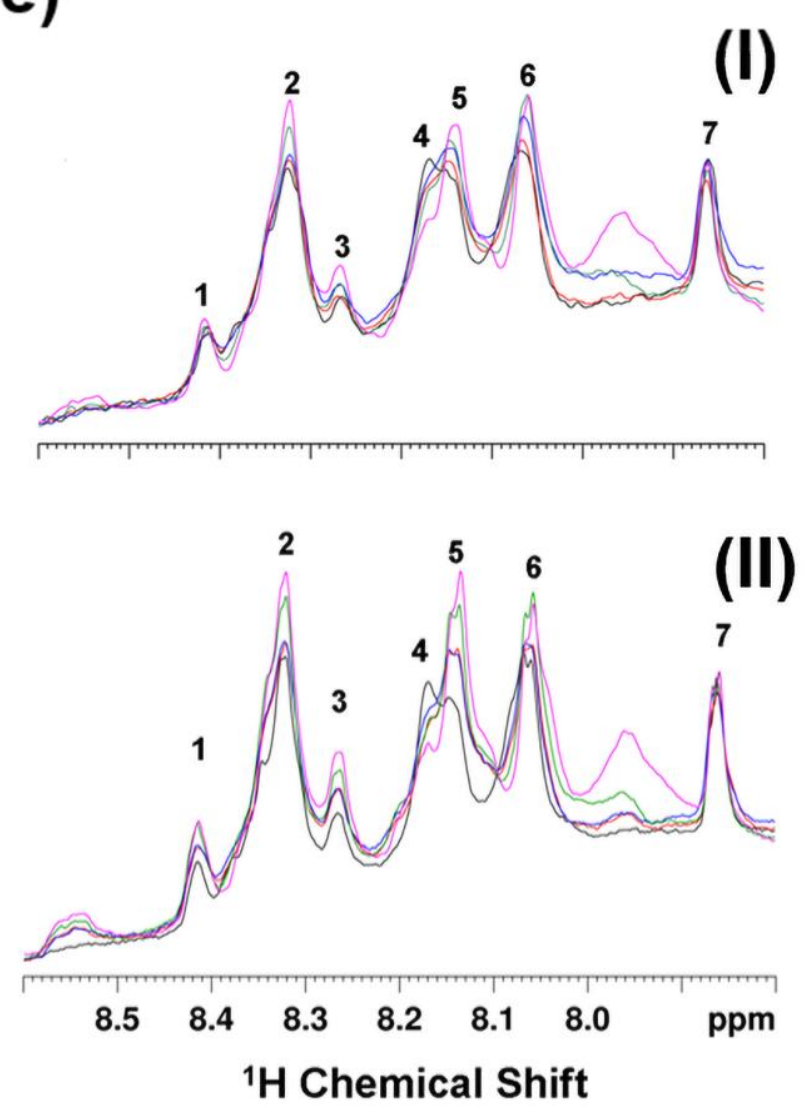

This article is protected by copyright. All rights reserved. 

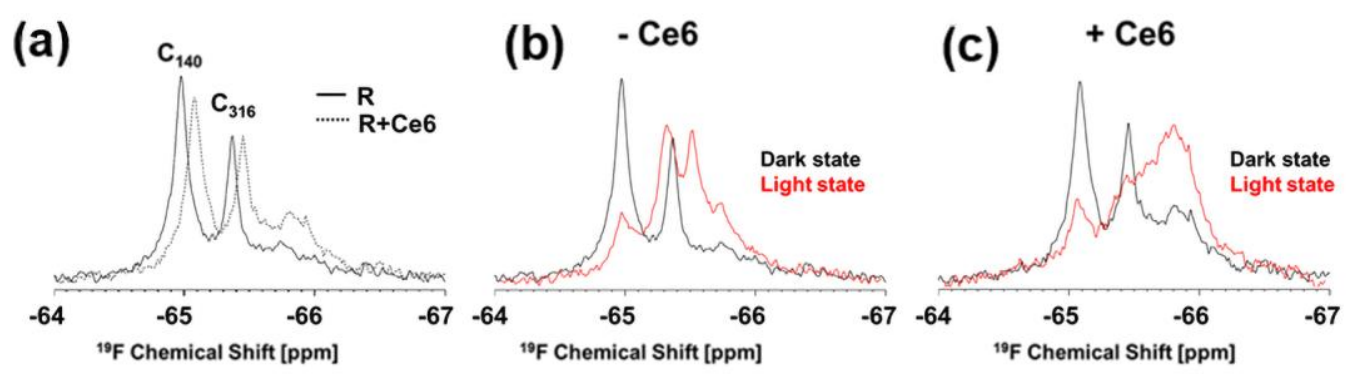

(d)

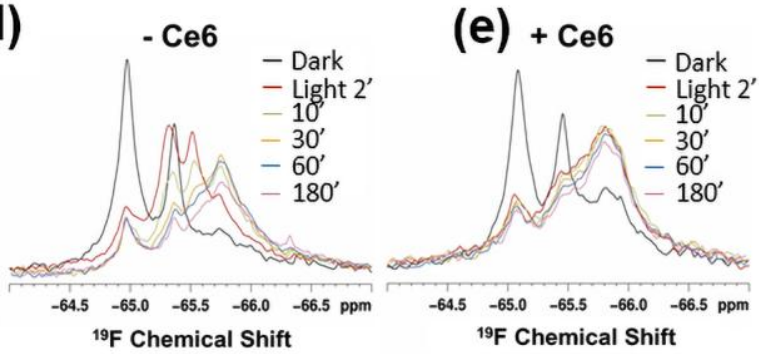

(f)

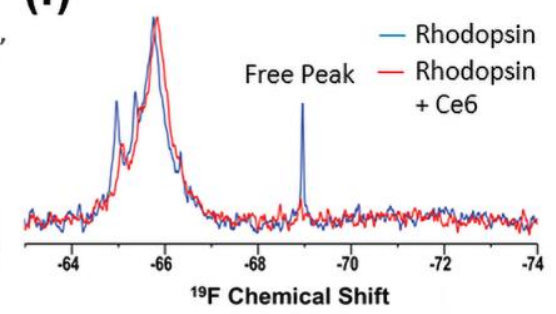

This article is protected by copyright. All rights reserved. 
(a)
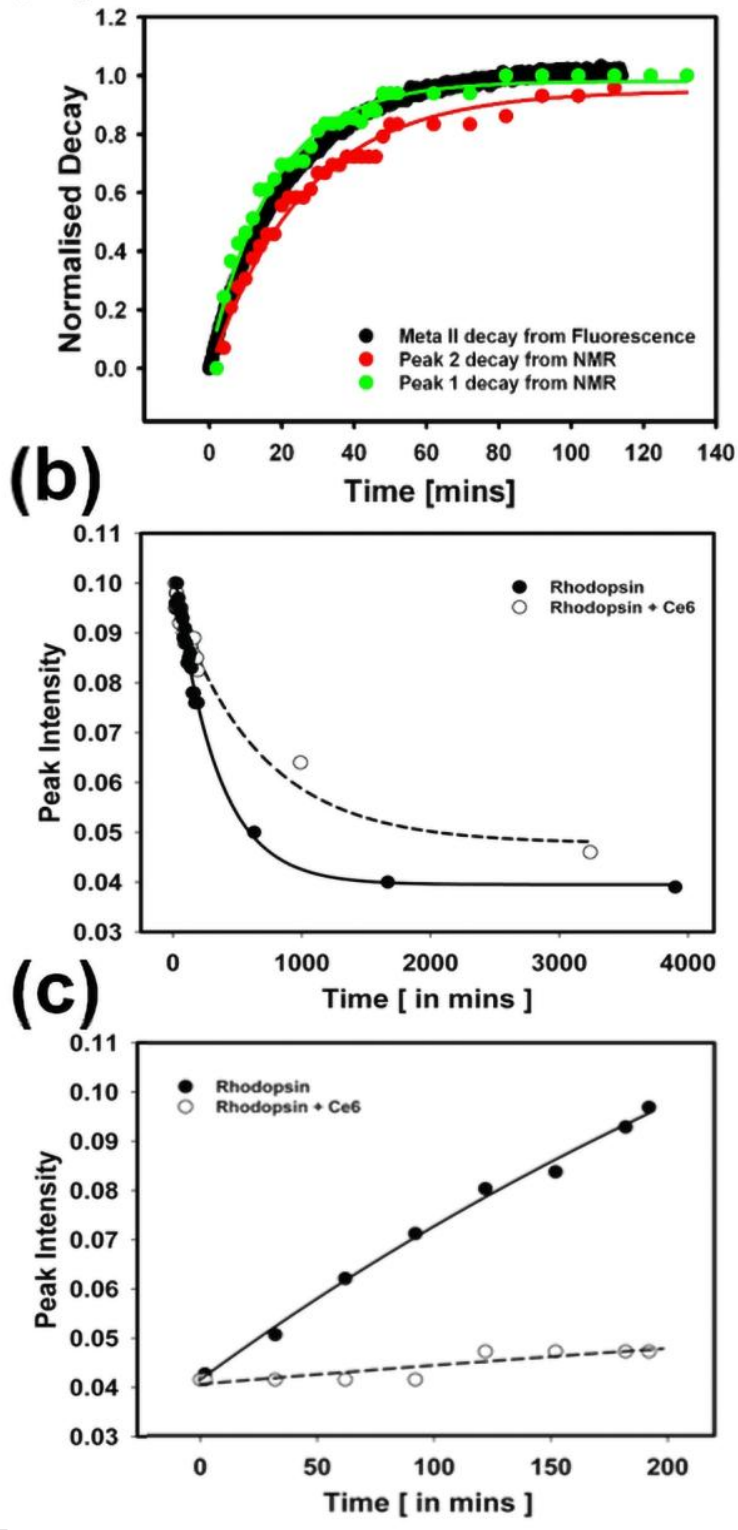

This article is protected by copyright. All rights reserved. 

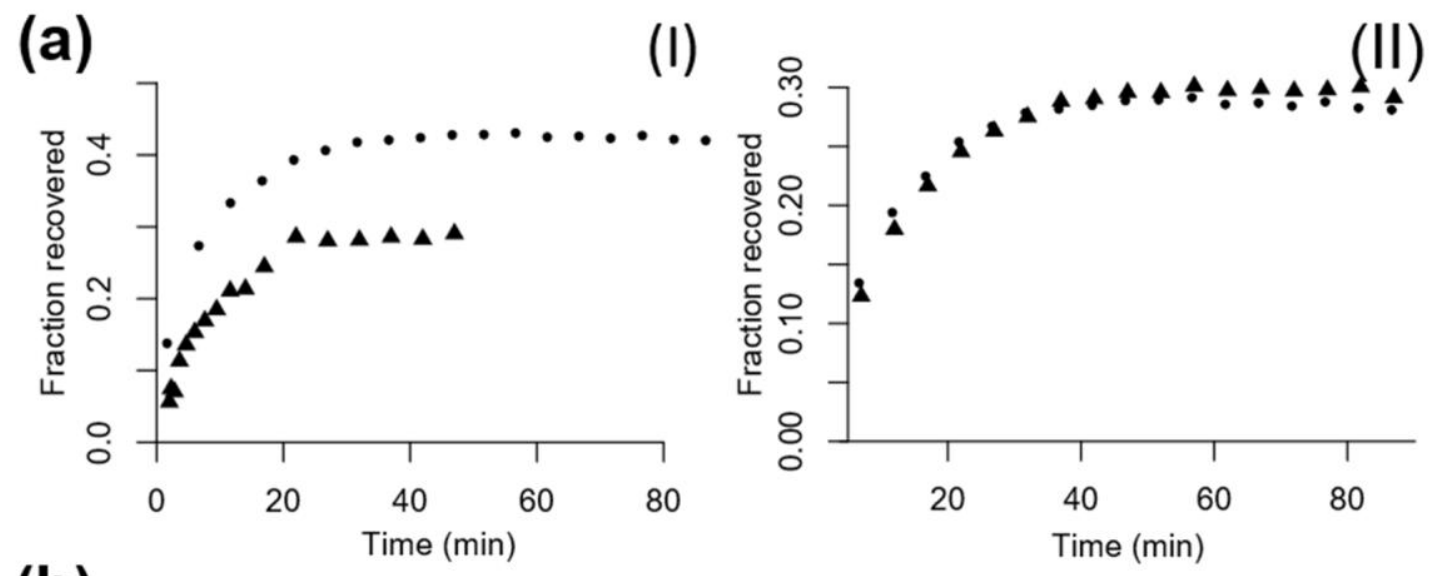

(b)

(I)
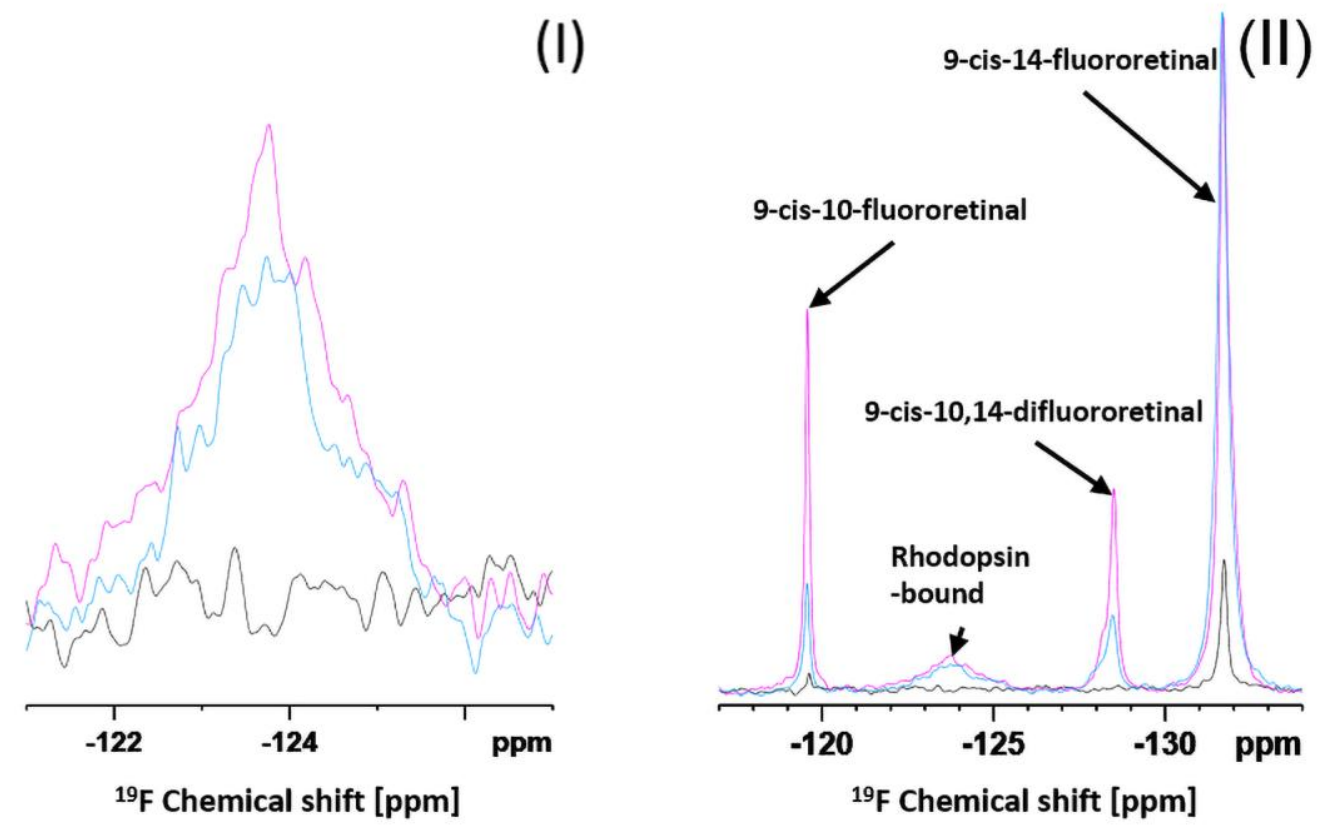

- $\mathbf{R}$
$-\mathbf{R}+10 \times \mathrm{Ce} 6$

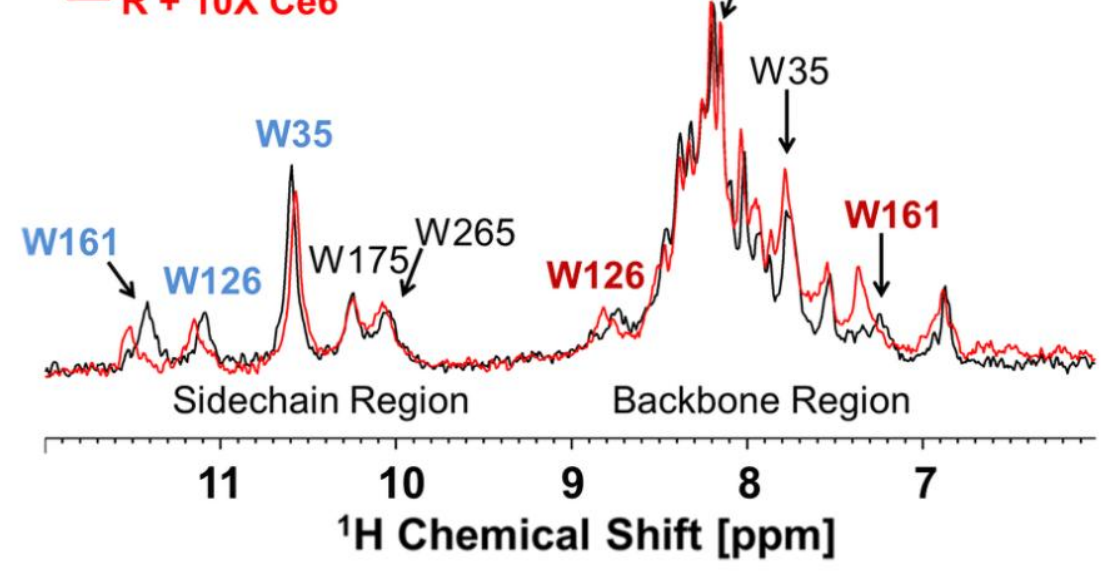

This article is protected by copyright. All rights reserved. 


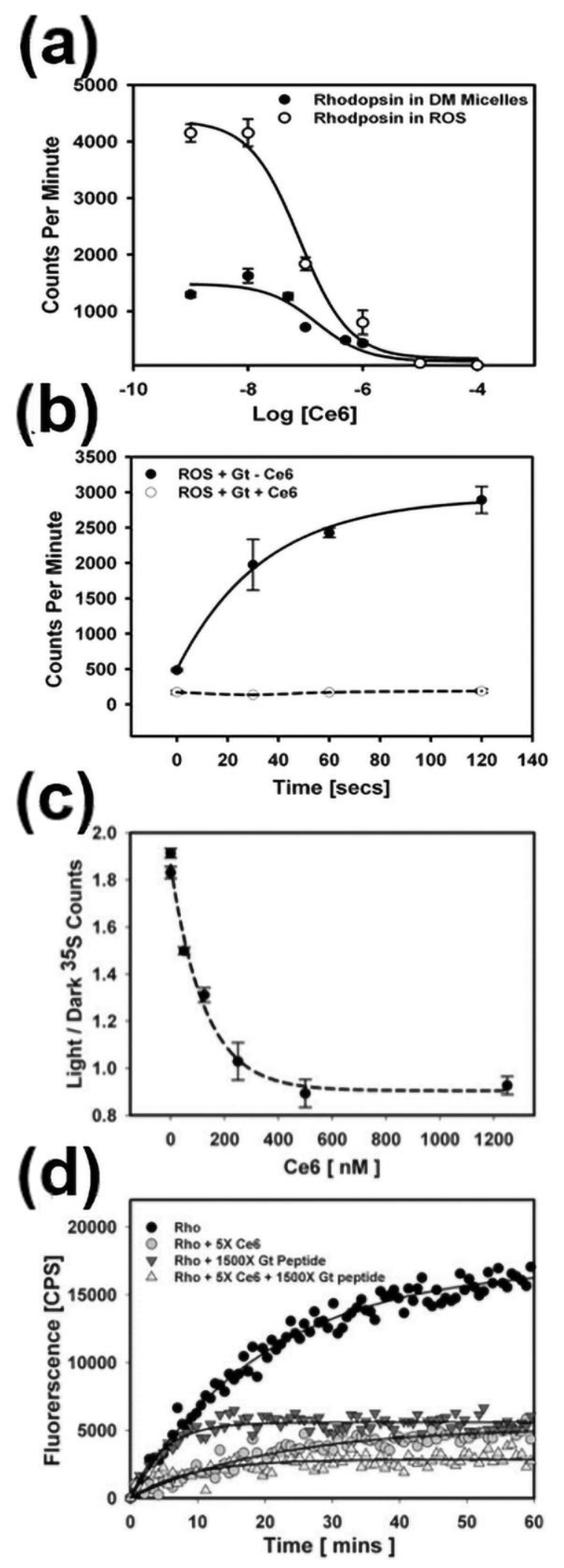

This article is protected by copyright. All rights reserved. 
(a)

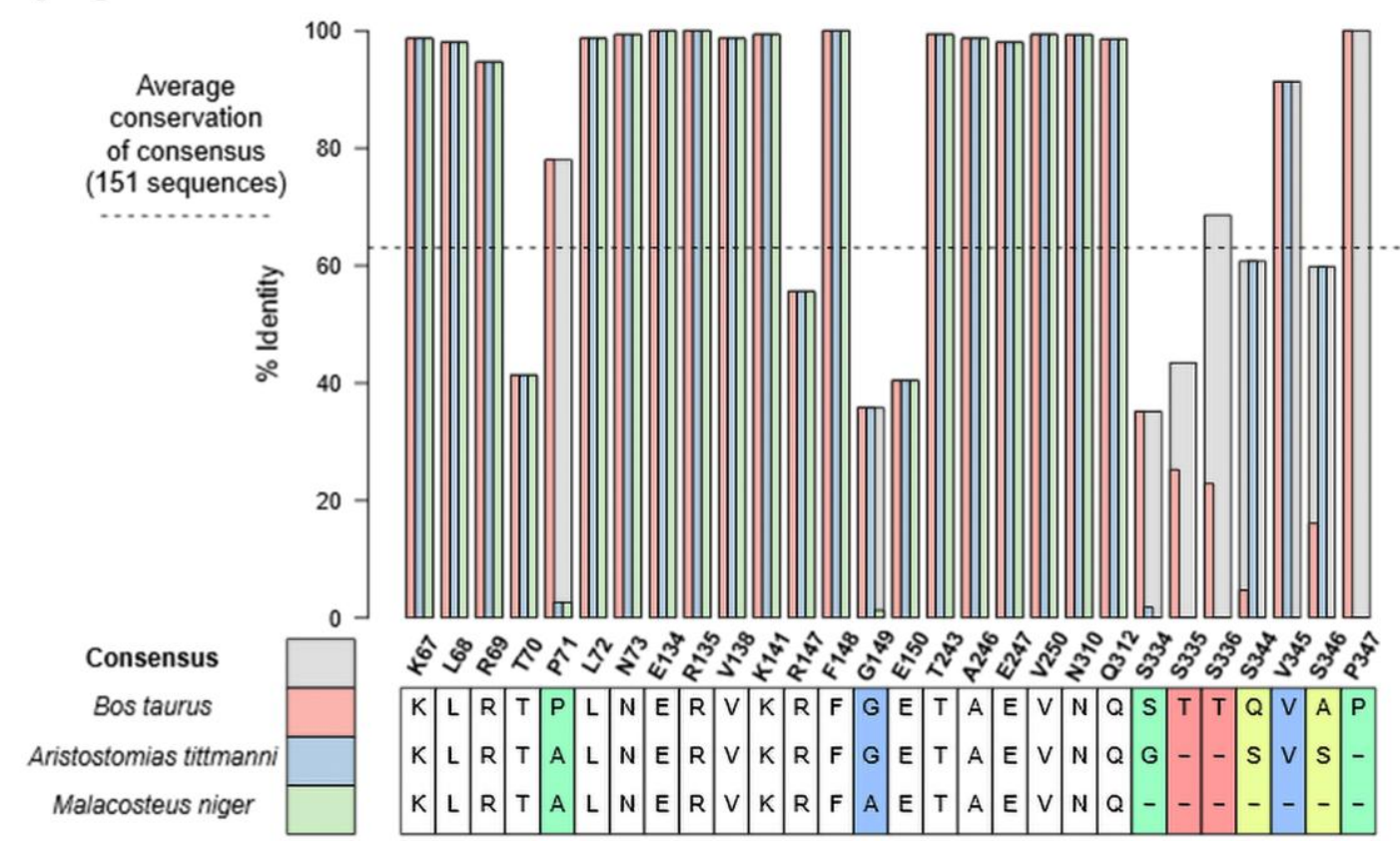

(b)

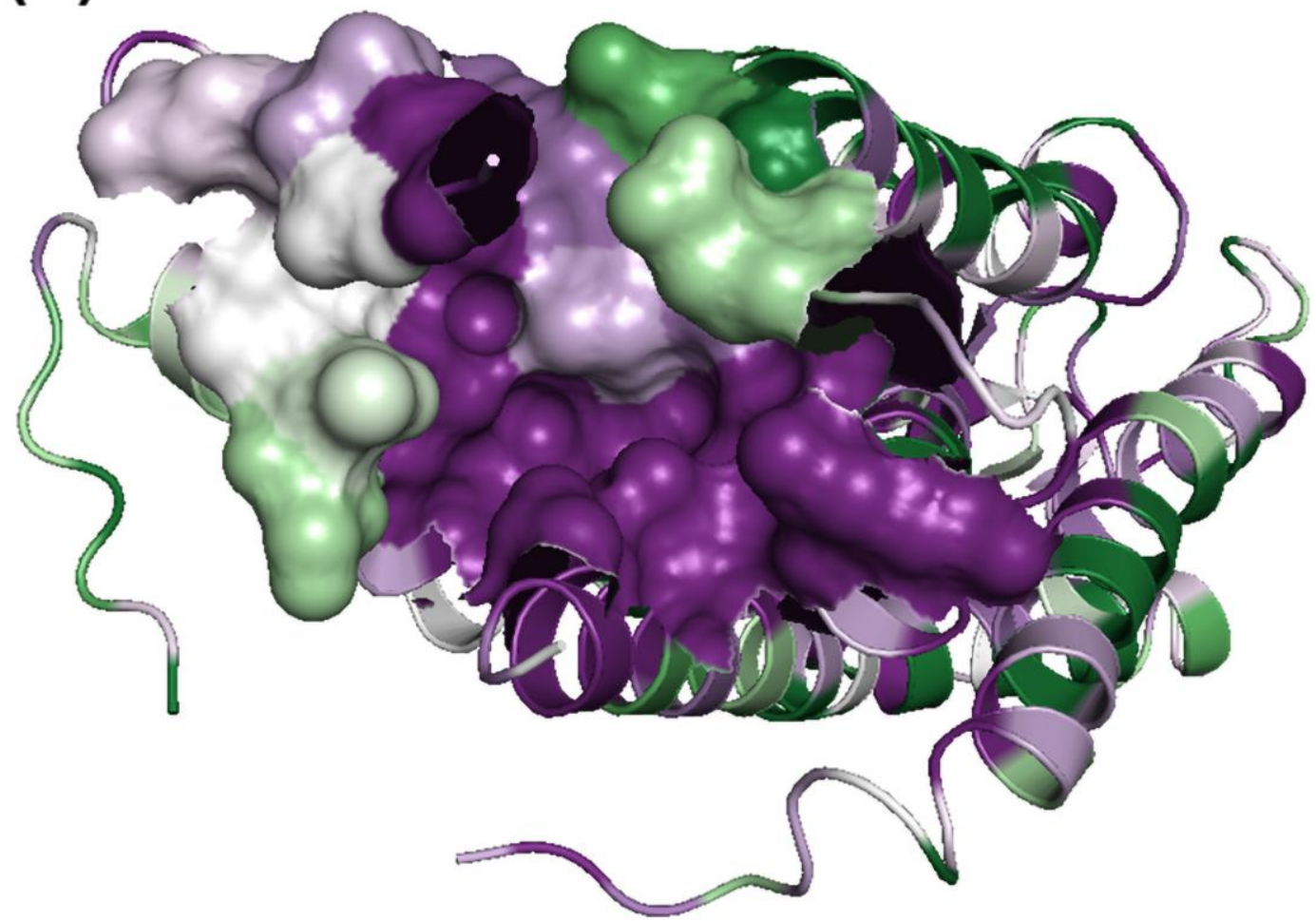
\begin{tabular}{l|l|l|l|l|l|l|l|l|}
1 & 2 & 3 & 4 & 5 & 6 & 7 & 8 & 9
\end{tabular}

Variable

Conserved

This article is protected by copyright. All rights reserved. 